\title{
A Class of Bridges of Iterated Integrals of Brownian Motion Related to Various Boundary Value Problems Involving the One-Dimensional Polyharmonic Operator
}

\author{
Aimé Lachal ${ }^{1,2}$ \\ ${ }^{1}$ Institut Camille Jordan, CNRS UMR5208, Université de Lyon, 20 avenue Albert Einstein, \\ 69621 Villeurbanne Cedex, France \\ ${ }^{2}$ Institut National des Sciences Appliquées de Lyon, Bâtiment Léonard de Vinci, \\ 20 avenue Albert Einstein, 69621 Villeurbanne Cedex, France
}

Correspondence should be addressed to Aimé Lachal, aime.lachal@insa-lyon.fr

Received 8 August 2011; Accepted 4 October 2011

Academic Editor: R. Liptser

Copyright (C) 2011 Aimé Lachal. This is an open access article distributed under the Creative Commons Attribution License, which permits unrestricted use, distribution, and reproduction in any medium, provided the original work is properly cited.

Let $(B(t))_{t \in[0,1]}$ be the linear Brownian motion and $\left(X_{n}(t)\right)_{t \in[0,1]}$ the $(n-1)$-fold integral of Brownian motion, with $n$ being a positive integer: $X_{n}(t)=\int_{0}^{t}\left((t-s)^{n-1} /(n-1) !\right) \mathrm{d} B(s)$ for any $t \in[0,1]$. In this paper we construct several bridges between times 0 and 1 of the process $\left(X_{n}(t)\right)_{t \in[0,1]}$ involving conditions on the successive derivatives of $X_{n}$ at times 0 and 1 . For this family of bridges, we make a correspondence with certain boundary value problems related to the one-dimensional polyharmonic operator. We also study the classical problem of prediction. Our results involve various Hermite interpolation polynomials.

\section{Introduction}

Throughout the paper, we will denote, for any enough differentiable function $f$, its $i$ th derivative by $f^{(i)}$ or $\mathrm{d}^{i} f / \mathrm{d} t^{i}$.

Let $(B(t))_{t \in[0,1]}$ be the linear Brownian motion started at 0 and $(\beta(t))_{t \in[0,1]}$ be the linear Brownian bridge within the time interval $[0,1]:(\beta(t))_{t \in[0,1]}=(B(t) \mid B(0)=B(1)=0)_{t \in[0,1]}$. These processes are Gaussian processes with covariance functions

$$
c_{B}(s, t)=s \wedge t, \quad c_{\beta}(s, t)=s \wedge t-s t .
$$


For a given continuous function $u$, the functions $v_{B}$ and $v_{\beta}$, respectively, defined on $[0,1]$ by

$$
v_{B}(t)=\int_{0}^{1} c_{B}(s, t) u(s) \mathrm{d} s, \quad v_{\beta}(t)=\int_{0}^{1} c_{\beta}(s, t) u(s) \mathrm{d} s
$$

are the solutions of the respective boundary value problems on $[0,1]$ :

$$
\left\{\begin{array} { l } 
{ v _ { B } ^ { \prime \prime } = - u , } \\
{ v _ { B } ( 0 ) = v _ { B } ^ { \prime } ( 1 ) = 0 , }
\end{array} \quad \left\{\begin{array}{l}
v_{\beta}^{\prime \prime}=-u, \\
v_{\beta}(0)=v_{\beta}(1)=0 .
\end{array}\right.\right.
$$

Observe that the differential equations are the same in both cases. Only the boundary conditions differ. They are Dirichlet-type boundary conditions for Brownian bridge while they are Dirichlet/Neumann-type boundary conditions for Brownian motion.

These well-known connections can be extended to the polyharmonic operator $\mathrm{d}^{2 n} / \mathrm{d} t^{2 n}$, where $n$ is a positive integer. This latter is associated with the $(n-1)$-fold integral of Brownian motion $\left(X_{n}(t)\right)_{t \in[0,1]}$ :

$$
X_{n}(t)=\int_{0}^{t} \frac{(t-s)^{n-1}}{(n-1) !} \mathrm{d} B(s) \quad \text { for any } t \in[0,1]
$$

(Notice that all of the derivatives at time 0 naturally vanish: $X_{n}(0)=X_{n-1}(0)=\cdots=X_{2}(0)=$ $X_{1}(0)=0$.) Indeed, the following facts, for instance, are known (see, e.g., $[1,2]$ ).

(i) The covariance function of the process $\left(X_{n}(t)\right)_{t \in[0,1]}$ coincides with the Green function of the boundary value problem

$$
\left\{\begin{array}{l}
v^{(2 n)}=(-1)^{n} u \quad \text { on }[0,1] \\
v(0)=v^{\prime}(0)=\cdots=v^{(n-1)}(0)=0 \\
v^{(n)}(1)=v^{(n+1)}(1)=\cdots=v^{(2 n-1)}(1)=0 .
\end{array}\right.
$$

(ii) The covariance fonction of the bridge $\left(X_{n}(t) \mid X_{n}(1)=0\right)_{t \in[0,1]}$ coincides with the Green function of the boundary value problem

$$
\left\{\begin{array}{l}
v^{(2 n)}=(-1)^{n} u \quad \text { on }[0,1] \\
v(0)=v^{\prime}(0)=\cdots=v^{(n-1)}(0)=0, \\
v^{(n-1)}(1)=v^{(n+1)}(1)=\cdots=v^{(2 n-1)}(1)=0 .
\end{array}\right.
$$


(iii) The covariance fonction of the bridge $\left(X_{n}(t) \mid X_{n}(1)=X_{n-1}(1)=\cdots=X_{1}(1)=\right.$ $0)_{t \in[0,1]}$ coincides with the Green function of the boundary value problem

$$
\left\{\begin{array}{l}
v^{(2 n)}=(-1)^{n} u \quad \text { on }[0,1], \\
v(0)=v^{\prime}(0)=\cdots=v^{(n-1)}(0)=0, \\
v(1)=v^{\prime}(1)=\cdots=v^{(n-1)}(1)=0 .
\end{array}\right.
$$

Observe that the differential equations and the boundary conditions at 0 are the same in all cases. Only the boundary conditions at 1 differ. Other boundary value problems can be found in $[3,4]$.

We refer the reader to [5] for a pioneering work dealing with the connections between general Gaussian processes and Green functions; see also [6]. We also refer to [7-13] and the references therein for various properties, namely, asymptotical study, of the iterated integrals of Brownian motion and more general Gaussian processes as well as to $[3,4,14,15]$ for interesting applications of these processes to statistics.

The aim of this work is to examine all the possible conditioned processes of $\left(X_{n}(t)\right)_{t \in[0,1]}$ involving different events at time 1:

$$
\left(X_{n}(t) \mid X_{j_{1}}(1)=X_{j_{2}}(1)=\cdots=X_{j_{m}}(1)=0\right)_{t \in[0,1]}
$$

for a certain number $m$ of events, $1 \leq m \leq n$, and certain indices $j_{1}, j_{2}, \ldots, j_{m}$ such that $1 \leq j_{1}<$ $j_{2}<\cdots<j_{m} \leq n$, and to make the connection with the boundary value problems:

$$
\left\{\begin{array}{l}
v^{(2 n)}=(-1)^{n} u \quad \text { on }[0,1], \\
v(0)=v^{\prime}(0)=\cdots=v^{(n-1)}(0)=0, \\
v^{\left(i_{1}\right)}(1)=v^{\left(i_{2}\right)}(1)=\cdots=v^{\left(i_{n}\right)}(1)=0
\end{array}\right.
$$

for certain indices $i_{1}, i_{2}, \ldots, i_{n}$ such that $0 \leq i_{1}<i_{2}<\cdots<i_{n} \leq 2 n-1$. Actually, we will see that this connection does not recover all the possible boundary value problems, and we will characterize those sets of indices for which such a connection exists.

The paper is organized as follows. In Section 2, we exhibit the relationships between general Gaussian processes and Green functions of certain boundary value problems. In Section 3, we consider the iterated integrals of Brownian motion. In Section 4, we construct several bridges associated with the foregoing processes and depict explicitly their connections with the polyharmonic operator together with various boundary conditions. One of the main results is Theorem 4.4. Moreover, we exhibit several interesting properties of the bridges (Theorems 4.1 and 4.3) and solve the prediction problem (Theorem 4.6). In Section 5, we illustrate the previous results on the case $n=2$ related to integrated Brownian motion. Finally, in Section 6, we give a characterization for the Green function of the boundary value problem (BVP) to be a covariance function. Another one of the main results is Theorem 6.3. 


\section{Gaussian Processes and Green Functions}

We consider an $n$-Markov Gaussian process $(X(t))_{t \in[0,1]}$ evolving on the real line $\mathbb{R}$. By " $n$ Markov," it is understood that the trajectory $t \mapsto X(t)$ is $n$ times differentiable and the $n$ dimensional process $\left(X(t), X^{\prime}(t), \ldots, X^{(n-1)}(t)\right)_{t \in[0,1]}$ is a Markov process. Let us introduce the covariance function of $(X(t))_{t \in[0,1]}$ : for $s, t \in[0,1], c_{X}(s, t)=\mathbb{E}[X(s) X(t)]$. It is known (see [6]) that the function $c_{X}$ admits the following representation:

$$
c_{X}(s, t)=\sum_{k=0}^{n-1} \varphi_{k}(s \wedge t) \psi_{k}(s \vee t)
$$

where $\varphi_{k}, \psi_{k}, k \in\{0,1, \ldots, n-1\}$, are certain functions.

Let $\Phi_{0}, \Phi_{1}$ be linear differential operators of order less than $p$, and let $\Phi$ be a linear differential operator of order $p$ defined by

$$
\boldsymbol{\Phi}=\sum_{i=0}^{p} \alpha_{i} \frac{\mathrm{d}^{i}}{\mathrm{~d} t^{i}}
$$

where $\alpha_{0}, \alpha_{1}, \ldots, \alpha_{p}$ are continuous functions on $[0,1]$. More precisely, we have for any $p$ times differentiable function $f$ and any $t \in[0,1]$,

$$
(\Phi f)(t)=\sum_{i=0}^{p} \alpha_{i}(t) f^{(i)}(t)
$$

Theorem 2.1. Assume that the functions $\varphi_{k}, \psi_{k}, k \in\{0,1, \ldots, n-1\}$, are $p$ times differentiable and satisfy the following conditions, for a certain constant $\kappa$ :

$$
\begin{gathered}
\sum_{k=0}^{n-1}\left[\varphi_{k} \psi_{k}^{(i)}-\varphi_{k}^{(i)} \psi_{k}\right]= \begin{cases}0 & \text { if } 0 \leq i \leq p-2, \\
\mathcal{K} & \text { if } i=p-1,\end{cases} \\
\Phi \varphi_{k}=\Phi \psi_{k}=0, \quad\left(\Phi_{0} \varphi_{k}\right)(0)=0, \quad\left(\Phi_{1} \psi_{k}\right)(1)=0 .
\end{gathered}
$$

Then, for any continuous function $u$ on $[0,1]$, the function $v$ defined on $[0,1]$ by

$$
v(t)=\int_{0}^{1} c_{X}(s, t) u(s) \mathrm{d} s
$$

solves the boundary value problem

$$
\left\{\begin{array}{l}
\boldsymbol{D}_{v}=\alpha_{p} u, \\
\left(\boldsymbol{\Phi}_{0} \boldsymbol{v}\right)(0)=\left(\boldsymbol{\Phi}_{1} \boldsymbol{v}\right)(1)=0 .
\end{array}\right.
$$

Remark 2.2. If the problem (2.7) is determining, that is, if it has a unique solution, then the covariance function $c_{X}$ is exactly the Green function of the boundary value problem (2.7). 
Proof. In view of (2.1), the function $v$ can be written as

$$
v(t)=\sum_{k=0}^{n-1}\left[\psi_{k}(t) \int_{0}^{t} \varphi_{k}(s) u(s) \mathrm{d} s+\varphi_{k}(t) \int_{t}^{1} \psi_{k}(s) u(s) \mathrm{d} s\right] .
$$

The derivative of $v$ is given by

$$
v^{\prime}(t)=\sum_{k=0}^{n-1}\left[\psi_{k}^{\prime}(t) \int_{0}^{t} \varphi_{k}(s) u(s) \mathrm{d} s+\varphi_{k}^{\prime}(t) \int_{t}^{1} \psi_{k}(s) u(s) \mathrm{d} s\right]
$$

and its second-order derivative, since $\sum_{k=0}^{n-1}\left[\varphi_{k} \psi_{k}^{\prime}-\varphi_{k}^{\prime} \psi_{k}\right]=0$, by

$$
\begin{aligned}
v^{\prime \prime}(t) & =\sum_{k=0}^{n-1}\left[\psi_{k}^{\prime \prime}(t) \int_{0}^{t} \varphi_{k}(s) u(s) \mathrm{d} s+\varphi_{k}^{\prime \prime}(t) \int_{t}^{1} \psi_{k}(s) u(s) \mathrm{d} s+\left[\varphi_{k}(t) \psi_{k}^{\prime}(t)-\varphi_{k}^{\prime}(t) \psi_{k}(t)\right] u(t)\right] \\
& =\sum_{k=0}^{n-1}\left[\psi_{k}^{\prime \prime}(t) \int_{0}^{t} \varphi_{k}(s) u(s) \mathrm{d} s+\varphi_{k}^{\prime \prime}(t) \int_{t}^{1} \psi_{k}(s) u(s) \mathrm{d} s\right]
\end{aligned}
$$

More generally, because of the assumptions (2.4), we easily see that, for $i \in\{0,1, \ldots, p-1\}$,

$$
v^{(i)}(t)=\sum_{k=0}^{n-1}\left[\psi_{k}^{(i)}(t) \int_{0}^{t} \varphi_{k}(s) u(s) \mathrm{d} s+\varphi_{k}^{(i)}(t) \int_{t}^{1} \psi_{k}(s) u(s) \mathrm{d} s\right]
$$

and the $p$ th order derivative of $v$ is given by

$$
\begin{aligned}
v^{(p)}(t)=\sum_{k=0}^{n-1}\left[\psi_{k}^{(p)}(t) \int_{0}^{t} \varphi_{k}(s) u(s) \mathrm{d} s+\varphi_{k}^{(p)}(t) \int_{t}^{1} \psi_{k}(s) u(s) \mathrm{d} s\right. \\
\left.+\left[\varphi_{k}(t) \psi_{k}^{(p-1)}(t)-\varphi_{k}^{(p-1)}(t) \psi(t)\right] u(t)\right] \\
=\sum_{k=0}^{n-1}\left[\psi_{k}^{(p)}(t) \int_{0}^{t} \varphi_{k}(s) u(s) \mathrm{d} s+\varphi_{k}^{(p)}(t) \int_{t}^{1} \psi_{k}(s) u(s) \mathrm{d} s\right]+\kappa u(t) .
\end{aligned}
$$

Actually, we have proved that, for $i \in\{0,1, \ldots, p-1\}$,

$$
v^{(i)}(t)=\int_{0}^{t} \frac{\partial^{i} c_{X}}{\partial t^{i}}(s, t) u(s) \mathrm{d} s
$$


Finally, due to (2.5),

$$
\Phi v(t)=\sum_{k=0}^{n-1}\left[\Phi \psi_{k}(t) \int_{0}^{t} \varphi_{k}(s) u(s) \mathrm{d} s+\Phi \varphi_{k}(t) \int_{t}^{1} \psi_{k}(s) u(s) \mathrm{d} s\right]+\kappa \alpha_{p} u(t)=\kappa \alpha_{p} u(t)
$$

Concerning the boundary value conditions, referring to (2.5), we similarly have

$$
\begin{aligned}
& \left(\Phi_{0} v\right)(0)=\sum_{k=0}^{n-1}\left(\Phi_{0} \varphi_{k}\right)(0) \int_{0}^{1} \psi_{k}(s) u(s) \mathrm{d} s=0, \\
& \left(\Phi_{1} v\right)(1)=\sum_{k=0}^{n-1}\left(\Phi_{1} \psi_{k}\right)(1) \int_{0}^{1} \varphi_{k}(s) u(s) \mathrm{d} s=0 .
\end{aligned}
$$

The proof of Theorem 2.1 is finished.

In the two next sections, we construct processes connected to the equation $\Phi v=u$ subject to the boundary value conditions at 0 : $\left(\boldsymbol{\Phi}_{0}^{i} v\right)(0)=0$ for $i \in\{0,1, \ldots, n-1\}$ and others at 1 that will be discussed subsequently, where $\Phi$ and $\Phi_{0}^{i}$ are the differential operators $(\Phi$ being of order $p=2 n$ ) defined by

$$
\Phi=(-1)^{n} \frac{\mathrm{d}^{2 n}}{\mathrm{~d} t^{2 n}}, \quad \Phi_{0}^{i}=\frac{\mathrm{d}^{i}}{\mathrm{~d} t^{i}}
$$

\section{The $(n-1)$-Fold Integral of Brownian Motion}

Let $(B(t))_{t \in[0,1]}$ be the linear Brownian motion limited to the time interval $[0,1]$ and started at 0 . We introduce the $(n-1)$-fold integral of Brownian motion: for any $t \in[0,1]$,

$$
X_{n}(t)=\int_{0}^{t} \frac{(t-s)^{n-1}}{(n-1) !} \mathrm{d} B(s)
$$

In particular, $X_{1}=B$. The trajectories of $\left(X_{n}(t)\right)_{t \in[0,1]}$ are $n$ times differentiable and we have $X_{n}^{(i)}=X_{n-i}$ for $0 \leq i \leq n-1$. Moreover, we have at time 0 the equalities $X_{n}(0)=X_{n-1}(0)=$ $\cdots=X_{2}(0)=X_{1}(0)=0$. The process $\left(X_{n}(t)\right)_{t \in[0,1]}$ is an $n$-Markov Gaussian process since the $n$-dimensional process $\left(X_{n}(t), X_{n-1}(t), \ldots, X_{1}(t)\right)_{t \in[0,1]}$ is Markovian. The covariance function of the Gaussian process $\left(X_{n}(t)\right)_{t \in[0,1]}$ is given by

$$
c_{X_{n}}(s, t)=\int_{0}^{s \wedge t} \frac{(s-u)^{n-1}}{(n-1) !} \frac{(t-u)^{n-1}}{(n-1) !} \mathrm{d} u .
$$


In order to apply Theorem 2.1, we decompose $c_{X_{n}}$ into the form (2.1). We have for, e.g., $s \leq t$,

$$
\begin{aligned}
c_{X_{n}}(s, t) & =\int_{0}^{s} \frac{(s-u)^{n-1}}{(n-1) !^{2}}\left[\sum_{k=0}^{n-1}\left(\begin{array}{c}
n-1 \\
k
\end{array}\right)(-u)^{n-1-k} t^{k}\right] \mathrm{d} u \\
& =\sum_{k=0}^{n-1}(-1)^{n-1-k} \frac{t^{k}}{k !} \int_{0}^{s} \frac{(s-u)^{n-1}}{(n-1) !} \frac{u^{n-1-k}}{(n-1-k) !} \mathrm{d} u \\
& =\sum_{k=0}^{n-1}(-1)^{n-1-k} \frac{s^{2 n-1-k}}{(2 n-1-k) !} \frac{t^{k}}{k !}
\end{aligned}
$$

We then obtain the following representation:

$$
c_{X_{n}}(s, t)=\sum_{k=0}^{n-1} \varphi_{k}(s) \psi_{k}(t)
$$

with, for any $k \in\{0,1, \ldots, n-1\}$,

$$
\varphi_{k}(s)=(-1)^{n-1-k} \frac{s^{2 n-1-k}}{(2 n-1-k) !}, \quad \psi_{k}(t)=\frac{t^{k}}{k !} .
$$

We state below a result of [2] that we revisit here by using Theorem 2.1.

Theorem 3.1. Let $u$ be a fixed continuous function on $[0,1]$. The function $v$ defined on $[0,1]$ by

$$
v(t)=\int_{0}^{1} c_{X_{n}}(s, t) u(s) \mathrm{d} s
$$

is the solution of the boundary value problem

$$
\left\{\begin{array}{l}
v^{(2 n)}=(-1)^{n} u \quad \text { on }[0,1] \\
v^{(i)}(0)=0 \text { for } i \in\{0,1, \ldots, n-1\} \\
v^{(i)}(1)=0 \text { for } i \in\{n, n+1, \ldots, 2 n-1\}
\end{array}\right.
$$


Proof. Let us check that conditions (2.4) and (2.5) of Theorem 2.1 are fulfilled. First, we have

$$
\begin{aligned}
& \sum_{k=0}^{n-1}\left[\varphi_{k}(t) \psi_{k}^{(i)}(t)-\varphi_{k}^{(i)}(t) \psi_{k}(t)\right] \\
& =\sum_{k=0}^{n-1}(-1)^{n-1-k}\left[\mathbb{1}_{\{k \geq i\}} \frac{t^{2 n-1-k}}{(2 n-1-k) !} \frac{t^{k-i}}{(k-i) !}-\mathbb{1}_{\{k \leq 2 n-1-i\}} \frac{t^{2 n-1-i-k}}{(2 n-1-i-k) !} \frac{t^{k}}{k !}\right] \\
& =(-1)^{n-1} \frac{t^{2 n-1-i}}{(2 n-1-i) !}\left[\mathbb{1}_{\{i \leq n-1\}} \sum_{k=i}^{n-1}(-1)^{k}\left(\begin{array}{c}
2 n-1-i \\
k-i
\end{array}\right)-\sum_{k=0}^{(2 n-1-i) \wedge(n-1)}(-1)^{k}\left(\begin{array}{c}
2 n-1-i \\
k
\end{array}\right)\right] \\
& =(-1)^{n-1} \frac{t^{2 n-1-i}}{(2 n-1-i) !}\left[\mathbb{1}_{\{i \leq n-1\}}\left(\sum_{k=0}^{n-1-i}(-1)^{i+k}\left(\begin{array}{c}
2 n-1-i \\
k
\end{array}\right)-\sum_{k=0}^{n-1}(-1)^{k}\left(\begin{array}{c}
2 n-1-i \\
k
\end{array}\right)\right)\right. \\
& \left.-\mathbb{1}_{\{i \geq n\}} \sum_{k=0}^{2 n-1-i}(-1)^{k}\left(\begin{array}{c}
2 n-1-i \\
k
\end{array}\right)\right] .
\end{aligned}
$$

Performing the transformation $k \mapsto 2 n-1-i-k$ in the first sum lying within the last equality, we get

$$
\begin{aligned}
& \sum_{k=0}^{n-1-i}(-1)^{i+k}\left(\begin{array}{c}
2 n-1-i \\
k
\end{array}\right)-\sum_{k=0}^{n-1}(-1)^{k}\left(\begin{array}{c}
2 n-1-i \\
k
\end{array}\right) \\
& \quad=\sum_{k=n}^{2 n-1-i}(-1)^{k}\left(\begin{array}{c}
2 n-1-i \\
2 n-1-i-k
\end{array}\right)+\sum_{k=0}^{n-1}(-1)^{k}\left(\begin{array}{c}
2 n-1-i \\
k
\end{array}\right)=\sum_{k=0}^{2 n-1-i}(-1)^{k}\left(\begin{array}{c}
2 n-1-i \\
k
\end{array}\right)=\delta_{i, 2 n-1},
\end{aligned}
$$

and then

$$
\sum_{k=0}^{n-1}\left[\varphi_{k}(t) \psi_{k}^{(i)}(t)-\varphi_{k}^{(i)}(t) \psi_{k}(t)\right]=(-1)^{n} \delta_{i, 2 n-1}
$$

On the other hand, setting

$$
\Phi=(-1)^{n} \frac{\mathrm{d}^{2 n}}{\mathrm{~d} t^{2 n}}, \quad \Phi_{0}^{i}=\frac{\mathrm{d}^{i}}{\mathrm{~d} t^{i}}, \quad \Phi_{1}^{i}=\frac{\mathrm{d}^{i+n}}{\mathrm{~d} t^{i+n}}
$$

we clearly see that, for any $k \in\{0,1, \ldots, n-1\}$,

$$
\Phi \varphi_{k}=\Phi \psi_{k}=0, \quad \Phi_{0}^{i} \varphi_{k}(0)=\Phi_{1}^{i} \psi_{k}(1)=0 \quad \text { for } i \in\{0,1, \ldots, n-1\}
$$

Consequently, by Theorem 2.1, we see that the function $v$ solves the boundary value problem (3.7). The uniqueness part will follow from a more general argument stated in the proof of Theorem 4.4 . 


\section{Various Bridges of the $(n-1)$-Fold Integral of Brownian Motion}

In this section, we construct various bridges related to $\left(X_{n}(t)\right)_{t \in[0,1]}$. More precisely, we take $\left(X_{n}(t)\right)_{t \in[0,1]}$ conditioned on the event that certain derivatives vanish at time 1 . Let us recall that all the derivatives at time 0 naturally vanish: $X_{n}(0)=X_{n-1}(0)=\cdots=X_{2}(0)=X_{1}(0)=0$.

For any $m \in\{0,1, \ldots, n\}$, let $J=\left\{j_{1}, j_{2}, \ldots, j_{m}\right\}$ be a subset of $\{1,2, \ldots, n\}$ with $1 \leq$ $j_{1}<j_{2}<\cdots<j_{m} \leq n$ and the convention that for $m=0, J=\emptyset$. We see that, for each $m \in\{0,1, \ldots, n\}$, we can define $\left(\begin{array}{c}n \\ m\end{array}\right)$ subsets of indices $J$, and the total number of sets $J$ is then $\sum_{m=0}^{n}\left(\begin{array}{c}n \\ m\end{array}\right)=2^{n}$. Set for any $t \in[0,1]$

$$
Y(t)=\left(X_{n}(t) \mid X_{j}(1)=0, j \in J\right)=\left(X_{n}(t) \mid X_{j_{1}}(1)=X_{j_{2}}(1)=\cdots=X_{j_{m}}(1)=0\right) .
$$

In this way, we define $2^{n}$ processes $(Y(t))_{t \in[0,1]}$ that we will call "bridges" of $\left(X_{n}(t)\right)_{t \in[0,1]}$. In particular,

(i) for $J=\emptyset$, we simply have $(Y(t))_{t \in[0,1]}=\left(X_{n}(t)\right)_{t \in[0,1]}$;

(ii) for $J=\{1\}$, the corresponding process is the $(n-1)$-fold integral of Brownian bridge

$$
\left(X_{n}(t) \mid X_{1}(1)=0\right)_{t \in[0,1]}=\left(\int_{0}^{t} \frac{(t-s)^{n-1}}{(n-1) !} \mathrm{d} \beta(s)\right)_{t \in[0,1]}
$$

(iii) for $J=\{n\}$, the corresponding process is the "single" bridge of $(n-1)$-fold integral of Brownian motion:

$$
\left(X_{n}(t) \mid X_{n}(1)=0\right)_{t \in[0,1]}=\left(\int_{0}^{t} \frac{(t-s)^{n-1}}{(n-1) !} \mathrm{d} B(s) \mid \int_{0}^{1} \frac{(1-s)^{n-1}}{(n-1) !} \mathrm{d} B(s)=0\right)_{t \in[0,1]} ;
$$

(iv) for $J=\{1,2, \ldots, n\}$, the corresponding process is

$$
\left(X_{n}(t) \mid X_{n}(1)=X_{n-1}(1)=\cdots=X_{1}(1)=0\right)_{t \in[0,1]} .
$$

This is the natural bridge related to the $n$-dimensional Markov process $\left(X_{n}(t), X_{n-1}(t), \ldots, X_{1}(t)\right)_{t \in[0,1]}$.

In this section, we exhibit several interesting properties of the various processes $(Y(t))_{t \in[0,1]}$. One of the main goals is to relate these bridges to additional boundary value conditions at 1 . For this, we introduce the following subset $I$ of $\{0,1, \ldots, 2 n-1\}$ :

$$
I=(n-J) \cup[\{n, n+1, \ldots, 2 n-1\} \backslash(J+n-1)]
$$

with

$$
\begin{gathered}
n-J=\{n-j, j \in J\}=\left\{n-j_{1}, \ldots, n-j_{m}\right\}, \\
J+n-1=\{j+n-1, j \in J\}=\left\{j_{1}+n-1, \ldots, j_{m}+n-1\right\} .
\end{gathered}
$$


Table 1

\begin{tabular}{ccccc}
\hline$I$ & $\{0,1, \ldots, n-1\}$ & $\{n, n+1, \ldots, 2 n-1\}$ & $\{n-1, n+1, \ldots, 2 n-1\}$ & $\{0, n+1, \ldots, 2 n-2\}$ \\
\hline$J$ & $\{1,2, \ldots, n\}$ & $\emptyset$ & $\{1\}$ & $\{n\}$ \\
\hline
\end{tabular}

The cardinality of $I$ is $n$. Actually, the set $I$ will be used further for enumerating the boundary value problems which can be related to the bridges labeled by $J$. Conversely, $I$ yields $J$ through

$$
J=(n-I) \cap\{1,2, \ldots, n\} .
$$

In Table 1, we give some examples of sets $I$ and $J$.

\subsection{Polynomial Drift Description}

Below, we provide a representation of $(Y(t))_{t \in[0,1]}$ by means of $\left(X_{n}(t)\right)_{t \in[0,1]}$ subject to a random polynomial drift.

Theorem 4.1. One has the distributional identity

$$
(Y(t))_{t \in[0,1]} \stackrel{d}{=}\left(X_{n}(t)-\sum_{j \in J} P_{j}(t) X_{j}(1)\right)_{t \in[0,1]},
$$

where the functions $P_{j}, j \in J$, are Hermite interpolation polynomials on $[0,1]$ characterized by

$$
\left\{\begin{array}{l}
P_{j}^{(2 n)}=0 \\
P_{j}^{(i)}(0)=0 \quad \text { for } i \in\{0,1, \ldots, n-1\} \\
P_{j}^{(i)}(1)=\delta_{j, n-i} \quad \text { for } i \in I .
\end{array}\right.
$$

Remark 4.2. In the case where $n=2$, we retrieve a result of [16]. Moreover, conditions (4.9) characterize the polynomials $P_{j}, j \in J$. We prove this fact in Lemma A.1 in the Appendix.

Proof. By invoking classical arguments of Gaussian processes theory, we have the distributional identity

$$
(Y(t))_{t \in[0,1]} \stackrel{d}{=}\left(X_{n}(t)-\sum_{j \in J} P_{j}(t) X_{j}(1)\right)_{t \in[0,1]},
$$

where the functions $P_{j}, j \in J$, are such that $\mathbb{E}\left[Y(t) X_{k}(1)\right]=0$ for all $k \in J$. We get the linear system

$$
\sum_{j \in J} \mathbb{E}\left[X_{j}(1) X_{k}(1)\right] P_{j}(t)=\mathbb{E}\left[X_{n}(t) X_{k}(1)\right], \quad k \in J
$$


We plainly have

$$
\mathbb{E}\left[X_{j}(s) X_{k}(t)\right]=\int_{0}^{s \wedge t} \frac{(s-u)^{j-1}}{(j-1) !} \frac{(t-u)^{k-1}}{(k-1) !} \mathrm{d} u .
$$

Then, the system (4.11) writes

$$
\sum_{j \in J} \frac{1}{j+k-1} \frac{P_{j}(t)}{(j-1) !}=\int_{0}^{t} \frac{(t-u)^{n-1}}{(n-1) !}(1-u)^{k-1} \mathrm{~d} u
$$

The matrix of this system $(1 /(j+k-1))_{j, k \in J}$ is regular as it can be seen by introducing the related quadratic form which is definite positive. Indeed, for any real numbers $x_{j}, j \in J$, we have

$$
\begin{gathered}
\sum_{j, k \in J} \frac{x_{j} x_{k}}{j+k-1}=\int_{0}^{1}\left(\sum_{j, k \in J} x_{j} x_{k} u^{j+k-2}\right) \mathrm{d} u=\int_{0}^{1}\left(\sum_{j \in J} x_{j} u^{j-1}\right)^{2} \mathrm{~d} u \geq 0, \\
\sum_{j, k \in J} \frac{x_{j} x_{k}}{j+k-1}=0 \quad \text { iff } \forall j \in J, x_{j}=0 .
\end{gathered}
$$

Thus, the system (4.11) has a unique solution. As a result, the $P_{j}$ are linear combinations of the functions $t \mapsto \int_{0}^{t}(t-u)^{n-1}(1-u)^{k-1} \mathrm{~d} u$ which are polynomials of degree less than $n+k$. Hence, the $P_{j}$ are polynomials of degree at most $2 n-1$.

We now compute the derivatives of $P_{j}$ at 0 in 1 . We have $P_{j}^{(i)}(0)=0$ for $i \in\{0,1, \ldots, n-$ $1\}$ since the functions $t \mapsto \int_{0}^{t}(t-u)^{n-1}(1-u)^{k-1} \mathrm{~d} u$ plainly enjoy this property. For checking that $P_{j}^{(i)}(1)=\delta_{j, n-i}$ for $i \in I$, we successively compute

(i) for $i \in\{0,1, \ldots, n-1\}$,

$$
\frac{\mathrm{d}^{i}}{\mathrm{~d} t^{i}} \mathbb{E}\left[X_{n}(t) X_{k}(1)\right]=\mathbb{E}\left[X_{n-i}(t) X_{k}(1)\right]
$$

(ii) for $i=n-1$,

$$
\frac{\mathrm{d}^{i}}{\mathrm{~d} t^{i}} \mathbb{E}\left[X_{n}(t) X_{k}(1)\right]=\mathbb{E}\left[B(t) X_{k}(1)\right]=\int_{0}^{t} \frac{(1-u)^{k-1}}{(k-1) !} \mathrm{d} u ;
$$

(iii) for $i=n$,

$$
\frac{\mathrm{d}^{i}}{\mathrm{~d} t^{i}} \mathbb{E}\left[X_{n}(t) X_{k}(1)\right]=\frac{(1-t)^{k-1}}{(k-1) !}
$$


(iv) for $i \in\{n, n+1, \ldots, n+k-1\}$,

$$
\frac{\mathrm{d}^{i}}{\mathrm{~d} t^{i}} \mathbb{E}\left[X_{n}(t) X_{k}(1)\right]=(-1)^{i+n} \frac{(1-t)^{k+n-i-1}}{(k+n-i-1) !}
$$

(v) for $i=k+n-1$,

$$
\frac{\mathrm{d}^{i}}{\mathrm{~d} t^{i}} \mathbb{E}\left[X_{n}(t) X_{k}(1)\right]=(-1)^{k-1}
$$

(vi) for $i \geq k+n$,

$$
\frac{\mathrm{d}^{i}}{\mathrm{~d} t^{i}} \mathbb{E}\left[X_{n}(t) X_{k}(1)\right]=0
$$

Consequently, at time $t=1$, for $i \geq n$,

$$
\left.\frac{\mathrm{d}^{i}}{\mathrm{~d} t^{i}} \mathbb{E}\left[X_{n}(t) X_{k}(1)\right]\right|_{t=1}=(-1)^{k-1} \delta_{i, k+n-1}
$$

Now, by differentiating (4.11), we get for $i \in\{0,1, \ldots, n-1\}$,

$$
\sum_{j \in J} \mathbb{E}\left[X_{j}(1) X_{k}(1)\right] P_{j}^{(i)}(1)=\mathbb{E}\left[X_{n-i}(1) X_{k}(1)\right], \quad k \in J .
$$

In particular, if $i \in(n-J)$, this can be rewritten as

$$
\sum_{j \in J} \mathbb{E}\left[X_{j}(1) X_{k}(1)\right] P_{j}^{(i)}(1)=\sum_{j \in J} \delta_{j, n-i} \mathbb{E}\left[X_{j}(1) X_{k}(1)\right], \quad k \in J,
$$

which by identification yields $P_{j}^{(i)}(1)=\delta_{j, n-i}$. Similarly, for $i \in\{n, n+1, \ldots, 2 n-1\}$, in view of (4.21), we have

$$
\sum_{j \in J} \mathbb{E}\left[X_{j}(1) X_{k}(1)\right] P_{j}^{(i)}(1)=(-1)^{k-1} \delta_{i, k+n-1}, \quad k \in J
$$

In particular, if $i \in\{n, n+1, \ldots, 2 n-1\} \backslash(J+n-1)$, we have $\delta_{i, k+n-1}=0$ for $k \in J$, and then

$$
\sum_{j \in J} \mathbb{E}\left[X_{j}(1) X_{k}(1)\right] P_{j}^{(i)}(1)=0, \quad k \in J
$$

which by identification yields $P_{j}^{(i)}(1)=0=\delta_{j, n-i}$. The proof of Theorem 4.1 is finished. 
International Journal of Stochastic Analysis

\subsection{Covariance Function}

Let $c_{Y}$ be the covariance function of $(Y(t))_{t \in[0,1]}: c_{Y}(s, t)=\mathbb{E}[Y(s) Y(t)]$. In the next theorem, we supply a representation of $c_{Y}$ of the form (2.1).

Theorem 4.3. The covariance function of $(Y(t))_{t \in[0,1]}$ admits the following representation: for $s, t \in$ $[0,1]$,

$$
c_{Y}(s, t)=\sum_{k=0}^{n-1} \varphi_{k}(s \wedge t) \tilde{\psi}_{k}(s \vee t)
$$

with, for any $k \in\{0,1, \ldots, n-1\}$,

$$
\widetilde{\psi}_{k}(t)=\psi_{k}(t)-\sum_{j \in J} \psi_{k}^{(n-j)}(1) P_{j}(t)
$$

Moreover, the functions $\widetilde{\psi}_{k}, k \in\{0,1, \ldots, n-1\}$, are Hermite interpolation polynomials such that

$$
\left\{\begin{array}{l}
\tilde{\psi}_{k}^{(2 n)}=0 \\
\tilde{\psi}_{k}^{(i)}(0)=\delta_{i, k} \quad \text { for } i \in\{0,1, \ldots, n-1\} \\
\tilde{\psi}_{k}^{(i)}(1)=0 \quad \text { for } i \in I .
\end{array}\right.
$$

Proof. We decompose $Y(t)$ into the difference

$$
Y(t)=X_{n}(t)-Z(t) \quad \text { with } Z(t)=\sum_{j \in J} P_{j}(t) X_{j}(1)
$$

We have

$$
\begin{aligned}
c_{Y}(s, t)= & \mathbb{E}\left[X_{n}(s) X_{n}(t)\right]+\mathbb{E}[Z(s) Z(t)]-\mathbb{E}\left[X_{n}(s) Z(t)\right]-\mathbb{E}\left[Z(s) X_{n}(t)\right] \\
= & c_{X_{n}}(s, t)+\sum_{j, k \in J} \mathbb{E}\left[X_{j}(1) X_{k}(1)\right] P_{j}(s) P_{k}(t) \\
& -\sum_{j \in J} \mathbb{E}\left[X_{n}(t) X_{j}(1)\right] P_{j}(s)-\sum_{j \in J} \mathbb{E}\left[X_{n}(s) X_{j}(1)\right] P_{j}(t) .
\end{aligned}
$$

By definition (4.11) of the $P_{j}{ }^{\prime}$ s, we observe that

$$
\begin{aligned}
& \sum_{j, k \in J} \mathbb{E} {\left[X_{j}(1) X_{k}(1)\right] P_{j}(s) P_{k}(t)-\sum_{k \in J} \mathbb{E}\left[X_{n}(s) X_{k}(1)\right] P_{k}(t) } \\
&=\sum_{k \in J}\left(\sum_{j \in J} \mathbb{E}\left[X_{j}(1) X_{k}(1)\right] P_{j}(s)-\mathbb{E}\left[X_{n}(s) X_{k}(1)\right]\right) P_{k}(t)=0 .
\end{aligned}
$$


Then, we can simplify $c_{Y}(s, t)$ into

$$
c_{Y}(s, t)=c_{X_{n}}(s, t)-\sum_{j \in J} \mathbb{E}\left[X_{n}(t) X_{j}(1)\right] P_{j}(s) .
$$

Since the covariance functions $c_{Y}$ and $c_{X_{n}}$ are symmetric, we also have

$$
c_{Y}(s, t)=c_{X_{n}}(s, t)-\sum_{j \in J} \mathbb{E}\left[X_{n}(s) X_{j}(1)\right] P_{j}(t)
$$

Let us introduce the symmetric polynomial

$$
Q(s, t)=\sum_{j \in J} \mathbb{E}\left[X_{n}(s) X_{j}(1)\right] P_{j}(t)=\sum_{j \in J} \mathbb{E}\left[X_{n}(t) X_{j}(1)\right] P_{j}(s) .
$$

It can be expressed by means of the functions $\varphi_{k}, \psi_{k}{ }^{\prime} \mathrm{s}$ as follows:

$$
Q(s, t)=\sum_{j \in J} \sum_{k=0}^{n-1} \varphi_{k}(s \wedge t) \psi_{k}^{(n-j)}(1) P_{j}(s \vee t)
$$

We can rewrite $c_{Y}(s, t)$ as

$$
c_{Y}(s, t)=c_{X_{n}}(s, t)-Q(s, t)=\sum_{k=0}^{n-1} \varphi_{k}(s \wedge t)\left[\psi_{k}(s \vee t)-\sum_{j \in J} \psi_{k}^{(n-j)}(1) P_{j}(s \vee t)\right],
$$

and then, for $s, t \in[0,1]$,

$$
c_{Y}(s, t)=\sum_{k=0}^{n-1} \varphi_{k}(s \wedge t) \tilde{\psi}_{k}(s \vee t) \quad \text { with } \tilde{\psi}_{k}(t)=\psi_{k}(t)-\sum_{j \in J} \psi_{k}^{(n-j)}(1) P_{j}(t)
$$

We immediately see that $\widetilde{\psi}_{k}$ is a polynomial of degree less than $2 n$ such that $\widetilde{\psi}_{k}^{(i)}(0)=\delta_{i, k}$ for $i \in\{0,1, \ldots, n-1\}$ and, since $P_{j}^{(i)}(1)=\delta_{j, n-i}$,

$$
\widetilde{\psi}_{k}^{(i)}(1)=\psi_{k}^{(i)}(1)-\sum_{j \in J} \psi_{k}^{(n-j)}(1) P_{j}^{(i)}(1)=\left(1-\mathbb{1}_{\{i \in n-J\}}\right) \psi_{k}^{(i)}(1)
$$

We deduce that

$$
\left\{\begin{array}{l}
\widetilde{\psi}_{k}^{(i)}(1)=0 \quad \text { if } i \in(n-J) \\
\widetilde{\psi}_{k}^{(i)}(1)=\psi_{k}^{(i)}(1)=0 \quad \text { if } i \in\{n, n+1, \ldots, 2 n-1\} \backslash(J+n-1)
\end{array}\right.
$$

Then $\widetilde{\psi}_{k}^{(i)}(1)=0$ for any $i \in I$. This ends up the proof of Theorem 4.3. 


\subsection{Boundary Value Problem}

In this section, we write out the natural boundary value problem which is associated with the process $(Y(t))_{t \in[0,1]}$. The following statement is the main connection between the different boundary value conditions associated with the operator $\mathrm{d}^{2 n} / \mathrm{d} t^{2 n}$ and the different bridges of the process $\left(X_{n}\right)_{t \in[0,1]}$ introduced in this work.

Theorem 4.4. Let $u$ be a fixed continuous function on $[0,1]$. The function $v$ defined on $[0,1]$ by

$$
v(t)=\int_{0}^{1} c_{Y}(s, t) u(s) \mathrm{d} s
$$

is the solution of the boundary value problem

$$
\left\{\begin{array}{l}
v^{(2 n)}=(-1)^{n} u \quad \text { on }[0,1] \\
v^{(i)}(0)=0 \text { for } i \in\{0,1, \ldots, n-1\} \\
v^{(i)}(1)=0 \text { for } i \in I .
\end{array}\right.
$$

Proof.

Step 1. Recall that $c_{Y}(s, t)=c_{X_{n}}(s, t)-Q(s, t)$. We decompose the function $v$ into the difference $w-z$, where, for $t \in[0,1]$,

$$
w(t)=\int_{0}^{1} c_{X_{n}}(s, t) u(s) \mathrm{d} s, \quad z(t)=\int_{0}^{1} Q(s, t) u(s) \mathrm{d} s .
$$

We know from Theorem 3.1 that $w^{(2 n)}=(-1)^{n} u, w^{(i)}(0)=\left(\partial^{i} Q / \partial t^{i}\right)(s, 0)=0$ for $i \in$ $\{0,1, \ldots, n-1\}$ and $w^{(i)}(1)=0$ for $i \in\{n, n+1, \ldots, 2 n-1\}$. Moreover, with the function $t \mapsto Q(s, t)$ being a polynomial of degree less than $2 n$, the function $z$ is also a polynomial of degree less than $2 n$. Then $z^{(2 n)}=0, z^{(i)}(0)=0$ for $i \in\{0,1, \ldots, n-1\}$, and

$$
v^{(2 n)}=w^{(2 n)}-z^{(2 n)}=(-1)^{n} u, \quad v^{(i)}(0)=w^{(i)}(0)-z^{(i)}(0)=0 \quad \text { for } i \in\{0,1, \ldots, n-1\}
$$

On the other hand, we learn from (2.13) that, for $i \in\{0,1, \ldots, 2 n-1\}$,

$$
w^{(i)}(1)=\int_{0}^{1} \frac{\partial^{i} c_{X_{n}}}{\partial t^{i}}(s, 1) u(s) \mathrm{d} s
$$

and then, for $i \in\{0,1, \ldots, n-1\}$,

$$
w^{(i)}(1)=\int_{0}^{1} \mathbb{E}\left[X_{n}(s) X_{n-i}(1)\right] u(s) \mathrm{d} s
$$


We also have, for any $i \in\{0,1, \ldots, 2 n-1\}$,

$$
\frac{\partial^{i} Q}{\partial t^{i}}(s, 1)=\sum_{j \in J} \mathbb{E}\left[X_{n}(s) X_{j}(1)\right] P_{j}^{(i)}(1)=\mathbb{1}_{\{i \in(n-J)\}} \mathbb{E}\left[X_{n}(s) X_{n-i}(1)\right]
$$

As a result, we see that

$$
z^{(i)}(1)=\int_{0}^{1} \frac{\partial^{i} Q}{\partial t^{i}}(s, 1) u(s) \mathrm{d} s=\mathbb{1}_{\{i \in(n-J)\}} w^{(i)}(1)
$$

This implies that, for $i \in(n-J), z^{(i)}(1)=w^{(i)}(1)$ and for $i \in\{n, n+1, \ldots, 2 n-1\} \backslash(J+n-1)$, $z^{(i)}(1)=0=w^{(i)}(1)$. Then $z^{(i)}(1)=w^{(i)}(1)$ for any $i \in I$, that is, $v^{(i)}(1)=0$. The function $v$ is a solution of (4.41).

Step 2. We now check the uniqueness of the solution of (4.41). Let $v_{1}$ and $v_{2}$ be two solutions of $\Phi v=u$. Then the function $w=v_{1}-v_{2}$ satisfies $\Phi w=0, w^{(i)}(0)=0$ for $i \in\{0,1, \ldots, n-1\}$ and $w^{(i)}(1)=0$ for $i \in I$. We compute the following "energy" integral:

$$
\begin{aligned}
\int_{0}^{1} w^{(n)}(t)^{2} \mathrm{~d} t & =(-1)^{n+1}\left[\sum_{i=0}^{n-1}(-1)^{i} w^{(i)}(t) w^{(2 n-1-i)}(t)\right]_{0}^{1}+(-1)^{n} \int_{0}^{1} w(t) w^{(2 n)}(t) \mathrm{d} t \\
& =(-1)^{n+1} \sum_{i=0}^{n-1}(-1)^{i} w^{(i)}(1) w^{(2 n-1-i)}(1) .
\end{aligned}
$$

We have constructed the set $I$ in order to have $w^{(i)}(1) w^{(2 n-1-i)}(1)=0$ for any $i \in\{0,1, \ldots, n-$ $1\}$ : when we pick an index $i \in\{0,1, \ldots, n-1\}$, either $i \in I$ or $2 n-1-i \in I$. Indeed,

(i) if $i \in I \cap\{0,1, \ldots, n-1\}, w^{(i)}=0$;

(ii) if $i \in\{0,1, \ldots, n-1\} \backslash I$, by observing that $\{0,1, \ldots, n-1\} \backslash I=\{0,1, \ldots, n-1\} \backslash(n-J)$, we have $2 n-1-i \in\{n, n+1, \ldots, 2 n-1\} \backslash(J+n-1)$. Since $\{n, n+1, \ldots, 2 n-1\} \backslash(J+n-1) \subset$ $I$, we see that $2 n-1-i \in I$ and then $w^{(2 n-1-i)}=0$.

Next, $\int_{0}^{1} w^{(n)}(t)^{2} \mathrm{~d} t=0$ which entails $w^{(n)}=0$, that is, $w$ is a polynomial of degree less than $n$. Moreover, with the boundary value conditions at 0 , we obtain $w=0$ or $v_{1}=v_{2}$.

The proof of Theorem 4.4 is finished.

Remark 4.5. We have seen in the previous proof that uniqueness is assured as soon as the boundary value conditions at 1 satisfy $w^{(i)}(1) w^{(2 n-1-i)}(1)=0$ for any $i \in\{0,1, \ldots, n-1\}$. These conditions are fulfilled when the set $I=\left\{i_{1}, i_{2}, \ldots, i_{n}\right\} \subset\{0,1, \ldots, 2 n-1\}$ is such that $i_{1} \in\{0,2 n-1\}, i_{2} \in\{1,2 n-2\}, i_{3} \in\{2,2 n-3\}, \ldots, i_{n} \in\{n-1, n\}$. This is equivalent to say that $I$ and $(2 n-1-I)$ make up a partition of $\{0,1, \ldots, 2 n-1\}$, or

$$
2 n-1-I=\{0,1, \ldots, 2 n-1\} \backslash I .
$$


In this manner, we get $2^{n}$ different boundary value problems which correspond to the $2^{n}$ different bridges we have constructed. We will see in Section 6 that the above identity concerning the differentiating set $I$ characterizes the possibility for the Green function of the boundary value problem (4.41) to be the covariance of a Gaussian process.

\subsection{Prediction}

Now, we tackle the problem of the prediction for the process $(Y(t))_{t \in[0,1]}$.

Theorem 4.6. Fix $t_{0} \in[0,1]$. The shifted process $\left(Y\left(t+t_{0}\right)\right)_{t \in\left[0,1-t_{0}\right]}$ admits the following representation:

$$
\left(Y\left(t+t_{0}\right)\right)_{t \in\left[0,1-t_{0}\right]}=\left(\tilde{Y}_{t_{0}}(t)+\sum_{i=0}^{n-1} Q_{i, t_{0}}(t) Y^{(i)}\left(t_{0}\right)\right)_{t \in\left[0,1-t_{0}\right]}
$$

where

$$
\tilde{Y}_{t_{0}}(t)=\tilde{X}_{n}(t)-\sum_{j \in J} \widetilde{P}_{j, t_{0}}(t) \tilde{X}_{j}\left(1-t_{0}\right)
$$

The process $\left(\tilde{X}_{n}(t)\right)_{t \in\left[0,1-t_{0}\right]}$ is a copy of $\left(X_{n}(t)\right)_{t \in\left[0,1-t_{0}\right]}$ which is independent of $\left(X_{n}(t)\right)_{t \in\left[0, t_{0}\right]}$.

The functions $\widetilde{P}_{j, t_{0}}, j \in J$, and $Q_{i, t_{0}}, i \in\{0,1, \ldots, n-1\}$, are Hermite interpolation polynomials on $\left[0,1-t_{0}\right]$ characterized by

$$
\begin{aligned}
& \left\{\begin{array}{l}
\tilde{P}_{j, t_{0}}^{(2 n)}=0, \\
\tilde{P}_{j, t_{0}}^{(\iota)}(0)=0 \quad \text { for } \iota \in\{0,1, \ldots, n-1\}, \\
\tilde{P}_{j, t_{0}}^{(\iota)}\left(1-t_{0}\right)=\delta_{\iota, n-j} \quad \text { for } \iota \in I,
\end{array}\right. \\
& \left\{\begin{array}{l}
Q_{i, t_{0}}^{(2 n)}=0, \\
Q_{i, t_{0}}^{(\iota)}(0)=\delta_{\iota, i} \quad \text { for } \iota \in\{0,1, \ldots, n-1\}, \\
Q_{i, t_{0}}^{(\iota)}\left(1-t_{0}\right)=0 \text { for } \iota \in I .
\end{array}\right.
\end{aligned}
$$

Actually, these functions can be expressed by means of the functions $P_{j}, j \in J$, and $\widetilde{\psi}_{i}, i \in\{0,1, \ldots, n-$ 1\}, as follows:

$$
\widetilde{P}_{j, t_{0}}(t)=\left(1-t_{0}\right)^{n-j} P_{j}\left(\frac{t}{1-t_{0}}\right), \quad Q_{i, t_{0}}=\left(1-t_{0}\right)^{i} \widetilde{\psi}_{i}\left(\frac{t}{1-t_{0}}\right)
$$

In other words, the process $\left(\tilde{Y}_{t_{0}}(t)\right)_{t \in\left[0,1-t_{0}\right]}$ is a bridge of length $\left(1-t_{0}\right)$ which is independent of $(Y(t))_{t \in\left[0, t_{0}\right]}$, that is,

$$
\left(\tilde{Y}_{t_{0}}(t)\right)_{t \in\left[0,1-t_{0}\right]} \stackrel{d}{=}\left(\tilde{X}_{n}(t) \mid \tilde{X}_{j}\left(1-t_{0}\right)=0, j \in J\right)_{t \in\left[0,1-t_{0}\right]}
$$


Proof.

Step 1. Fix $t_{0} \in[0,1]$. We have the well-known decomposition, based on the classical prediction property of Brownian motion stipulating that $\left(B\left(t+t_{0}\right)\right)_{t \in\left[0,1-t_{0}\right]}=\left(\widetilde{B}_{t_{0}}(t)+\right.$ $\left.B\left(t_{0}\right)\right)_{t \in\left[0,1-t_{0}\right]}$, where $\left(\widetilde{B}_{t_{0}}(t)\right)_{t \in\left[0,1-t_{0}\right]}$ is a Brownian motion independent of $(B(t))_{t \in\left[0, t_{0}\right]}$,

$$
\left(X_{n}\left(t+t_{0}\right)\right)_{t \in\left[0,1-t_{0}\right]}=\left(\tilde{X}_{n}(t)+\sum_{k=0}^{n-1} \frac{t^{k}}{k !} X_{n-k}\left(t_{0}\right)\right)_{t \in\left[0,1-t_{0}\right]}
$$

with $\tilde{X}_{n}(t)=\int_{0}^{1}\left((t-s)^{n-1} /(n-1) !\right) \mathrm{d} \widetilde{B}(s)$. Differentiating this equality $(n-j)$ times, $j \in$ $\{1,2, \ldots, n\}$, we obtain

$$
X_{j}\left(t+t_{0}\right)=\tilde{X}_{j}(t)+\sum_{k=n-j}^{n-1} \frac{t^{j+k-n}}{(j+k-n) !} X_{n-k}\left(t_{0}\right)
$$

Therefore,

$$
\begin{aligned}
Y\left(t+t_{0}\right)= & X_{n}\left(t+t_{0}\right)-\sum_{j \in J} P_{j}\left(t+t_{0}\right) X_{j}(1) \\
= & \tilde{X}_{n}(t)+\sum_{k=0}^{n-1} \frac{t^{k}}{k !} X_{n-k}\left(t_{0}\right)-\sum_{j \in J} P_{j}\left(t+t_{0}\right)\left[\tilde{X}_{j}\left(1-t_{0}\right)+\sum_{k=n-j}^{n-1} \frac{\left(1-t_{0}\right)^{j+k-n}}{(j+k-n) !} X_{n-k}\left(t_{0}\right)\right] \\
= & {\left[\tilde{X}_{n}(t)-\sum_{j \in J} P_{j}\left(t+t_{0}\right) \tilde{X}_{j}\left(1-t_{0}\right)\right] } \\
& +\sum_{k=0}^{n-1}\left[\frac{t^{k}}{k !}-\sum_{j \in J: j \geq n-k} \frac{\left(1-t_{0}\right)^{j+k-n}}{(j+k-n) !} P_{j}\left(t+t_{0}\right)\right] X_{n-k}\left(t_{0}\right) .
\end{aligned}
$$

We are going to express the $X_{n-k}\left(t_{0}\right), k \in\{1, \ldots, n\}$, by means of the $\tilde{X}_{j}\left(1-t_{0}\right), j \in J$. We have, by differentiating (4.57) $i$ times, for $i \in\{0,1, \ldots, n-1\}$,

$$
\begin{aligned}
Y^{(i)}\left(t+t_{0}\right)= & {\left[\tilde{X}_{n-i}(t)-\sum_{j \in J} P_{j}^{(i)}\left(t+t_{0}\right) \tilde{X}_{j}\left(1-t_{0}\right)\right] } \\
& +\sum_{k=0}^{n-1}\left[\frac{t^{k-i}}{(k-i) !} \mathbb{1}_{\{k \geq i\}}-\sum_{j \in J: j \geq n-k} \frac{\left(1-t_{0}\right)^{j+k-n}}{(j+k-n) !} P_{j}^{(i)}\left(t+t_{0}\right)\right] X_{n-k}\left(t_{0}\right) .
\end{aligned}
$$


For $t=0$, this yields

$$
Y^{(i)}\left(t_{0}\right)=-\sum_{j \in J} P_{j}^{(i)}\left(t_{0}\right) \tilde{X}_{j}\left(1-t_{0}\right)+\sum_{k=0}^{n-1}\left[\delta_{i, k}-\sum_{j \in J: j \geq n-k} \frac{\left(1-t_{0}\right)^{j+k-n}}{(j+k-n) !} P_{j}^{(i)}\left(t_{0}\right)\right] X_{n-k}\left(t_{0}\right) .
$$

Set for $i, k \in\{0,1, \ldots, n-1\}$

$$
a_{i k}=\delta_{i, k}-\sum_{j \in J: j \geq n-k} \frac{\left(1-t_{0}\right)^{j+k-n}}{(j+k-n) !} P_{j}^{(i)}\left(t_{0}\right),
$$

and let us introduce the matrix

$$
A=\left(a_{i k}\right)_{0 \leq i, k \leq n-1}
$$

together with its inverse matrix

$$
B=A^{-1}=\left(b_{i k}\right)_{0 \leq i, k \leq n-1} .
$$

Equalities (4.59) for $i \in\{0,1, \ldots, n-1\}$ read as a linear system of $(n-1)$ equations and $(n-1)$ unknowns:

$$
\sum_{k=0}^{n-1} a_{i k} X_{n-k}\left(t_{0}\right)=Y^{(i)}\left(t_{0}\right)+\sum_{j \in J} P_{j}^{(i)}\left(t_{0}\right) \tilde{X}_{j}\left(1-t_{0}\right), \quad i \in\{0,1, \ldots, n-1\},
$$

which can be rewritten into a matrix form as

$$
A\left(\begin{array}{c}
X_{n}\left(t_{0}\right) \\
X_{n-1}\left(t_{0}\right) \\
\vdots \\
X_{2}\left(t_{0}\right) \\
X_{1}\left(t_{0}\right)
\end{array}\right)=\left(\begin{array}{c}
Y\left(t_{0}\right) \\
Y^{\prime}\left(t_{0}\right) \\
\vdots \\
Y^{(n-2)}\left(t_{0}\right) \\
Y^{(n-1)}\left(t_{0}\right)
\end{array}\right)+\sum_{j \in J} \tilde{X}_{j}\left(1-t_{0}\right)\left(\begin{array}{c}
P_{j}\left(t_{0}\right) \\
P_{j}^{\prime}\left(t_{0}\right) \\
\vdots \\
P_{j}^{(n-2)}\left(t_{0}\right) \\
P_{j}^{(n-1)}\left(t_{0}\right)
\end{array}\right) .
$$

The solution is given by

$$
\left(\begin{array}{c}
X_{n}\left(t_{0}\right) \\
X_{n-1}\left(t_{0}\right) \\
\vdots \\
X_{2}\left(t_{0}\right) \\
X_{1}\left(t_{0}\right)
\end{array}\right)=B\left(\begin{array}{c}
Y\left(t_{0}\right) \\
Y^{\prime}\left(t_{0}\right) \\
\vdots \\
Y^{(n-2)}\left(t_{0}\right) \\
Y^{(n-1)}\left(t_{0}\right)
\end{array}\right)+\sum_{j \in J} \tilde{X}_{j}\left(1-t_{0}\right) B\left(\begin{array}{c}
P_{j}\left(t_{0}\right) \\
P_{j}^{\prime}\left(t_{0}\right) \\
\vdots \\
P_{j}^{(n-2)}\left(t_{0}\right) \\
P_{j}^{(n-1)}\left(t_{0}\right)
\end{array}\right)
$$


and we see that $X_{n-k}\left(t_{0}\right), k \in\{0,1, \ldots, n-1\}$, is of the form

$$
X_{n-k}\left(t_{0}\right)=\sum_{i=0}^{n-1} b_{k i} Y^{(i)}\left(t_{0}\right)+\sum_{j \in J}\left[\sum_{i=0}^{n-1} b_{k i} P_{j}^{(i)}\left(t_{0}\right)\right] \tilde{X}_{j}\left(1-t_{0}\right) .
$$

Therefore, by plugging (4.66) into (4.57), we obtain

$$
\begin{aligned}
& Y\left(t+t_{0}\right) \\
& =\tilde{X}_{n}(t)-\sum_{j \in J} P_{j}\left(t+t_{0}\right) \tilde{X}_{j}\left(1-t_{0}\right)+\sum_{k=0}^{n-1}\left[\frac{t^{k}}{k !}-\sum_{j \in J: j \geq n-k} \frac{\left(1-t_{0}\right)^{j+k-n}}{(j+k-n) !} P_{j}\left(t+t_{0}\right)\right] \\
& \quad \times\left[\sum_{i=0}^{n-1} b_{k i} Y^{(i)}\left(t_{0}\right)+\sum_{j \in J}\left(\sum_{i=0}^{n-1} b_{k i} P_{j}^{(i)}\left(t_{0}\right)\right) \tilde{X}_{j}\left(1-t_{0}\right)\right] \\
& =\tilde{X}_{n}(t)-\sum_{j \in J}\left[P_{j}\left(t+t_{0}\right)-\sum_{0 \leq i, k \leq n-1} b_{k i} P_{j}^{(i)}\left(t_{0}\right)\left(\frac{t^{k}}{k !}-\sum_{j \in J: j \geq n-k} \frac{\left(1-t_{0}\right)^{j+k-n}}{(j+k-n) !} P_{j}\left(t+t_{0}\right)\right)\right] \tilde{X}_{j}\left(1-t_{0}\right) \\
& \quad+\sum_{0 \leq i, k \leq n-1} b_{k i}\left[\frac{t^{k}}{k !}-\sum_{j \in J: j \geq n-k} \frac{\left(1-t_{0}\right)^{j+k-n}}{(j+k-n) !} P_{j}\left(t+t_{0}\right)\right] Y^{(i)}\left(t_{0}\right) .
\end{aligned}
$$

Finally, $Y\left(t+t_{0}\right)$ can be written as

$$
Y\left(t+t_{0}\right)=\tilde{Y}_{t_{0}}(t)+\sum_{i=0}^{n-1} Q_{i, t_{0}}(t) Y^{(i)}\left(t_{0}\right)
$$

where

$$
\begin{gathered}
Q_{i, t_{0}}(t)=\sum_{k=0}^{n-1} b_{k i}\left[\frac{t^{k}}{k !}-\sum_{j \in J: j \geq n-k} \frac{\left(1-t_{0}\right)^{j+k-n}}{(j+k-n) !} P_{j}\left(t+t_{0}\right)\right], \\
\tilde{Y}_{t_{0}}(t)=\tilde{X}_{n}(t)-\sum_{j \in J} \widetilde{P}_{j, t_{0}}(t) \tilde{X}_{j}\left(1-t_{0}\right)
\end{gathered}
$$

with

$$
\begin{aligned}
\widetilde{P}_{j, t_{0}}(t) & =P_{j}\left(t+t_{0}\right)-\sum_{0 \leq i, k \leq n-1} b_{k i} P_{j}^{(i)}\left(t_{0}\right)\left[\frac{t^{k}}{k !}-\sum_{j \in J: j \geq n-k} \frac{\left(1-t_{0}\right)^{j+k-n}}{(j+k-n) !} P_{j}\left(t+t_{0}\right)\right] \\
& =P_{j}\left(t+t_{0}\right)-\sum_{i=0}^{n-1} P_{j}^{(i)}\left(t_{0}\right) Q_{i, t_{0}}(t) .
\end{aligned}
$$


Step 2. We easily see that the functions $\widetilde{P}_{j, t_{0}}$ and $Q_{i, t_{0}}$ are polynomials of degree less than $2 n$. Let us compute now their derivatives at 0 and $t_{0}$. First, concerning $Q_{i, t_{0}}$ we have

$$
Q_{i, t_{0}}^{(\iota)}(t)=\sum_{k=0}^{n-1} b_{k i}\left[\frac{t^{k-\iota}}{(k-\iota) !} \mathbb{1}_{\{k \geq \imath\}}-\sum_{j \in J: j \geq n-k} \frac{\left(1-t_{0}\right)^{j+k-n}}{(j+k-n) !} P_{j}^{(\iota)}\left(t+t_{0}\right)\right] .
$$

Choosing $t=0$ and recalling the definition of $a_{t k}$ and the fact that the matrices $\left(a_{i k}\right)_{0 \leq i, k \leq n}$ and $\left(b_{i k}\right)_{0 \leq i, k \leq n}$ are inverse, this gives, for $\iota \in\{0,1, \ldots, n-1\}$,

$$
Q_{i, t_{0}}^{(\iota)}(0)=\sum_{k=0}^{n-1} b_{k i}\left[\delta_{\iota, k}-\sum_{j \in J: j \geq n-k} \frac{\left(1-t_{0}\right)^{j+k-n}}{(j+k-n) !} P_{j}^{(\iota)}\left(t_{0}\right)\right]=\sum_{k=0}^{n-1} a_{\iota k} b_{k i}=\delta_{\iota, i} .
$$

Choosing $t=1-t_{0}$, we have for $\iota \in I$

$$
Q_{i, t_{0}}^{(\iota)}\left(1-t_{0}\right)=\sum_{k=0}^{n-1} b_{k i}\left[\frac{\left(1-t_{0}\right)^{k-\iota}}{(k-\iota) !} \mathbb{1}_{\{k \geq \imath\}}-\sum_{j \in J: j \geq n-k} \frac{\left(1-t_{0}\right)^{j+k-n}}{(j+k-n) !} P_{j}^{(\iota)}(1)\right] .
$$

By Theorem 4.1, we know that $P_{j}^{(\iota)}(1)=\delta_{j, n-\iota}$ for $\iota \in I$; then

$$
\sum_{j \in J: j \geq n-k} \frac{\left(1-t_{0}\right)^{j+k-n}}{(j+k-n) !} P_{j}^{(\iota)}(1)=\frac{\left(1-t_{0}\right)^{k-\iota}}{(k-\iota) !} \mathbb{1}_{\{k \geq \iota\}} \mathbb{1}_{\{\ell \in(n-J)\}} .
$$

Observing that, if $\iota \leq k \leq n-1$, the conditions $\iota \in(n-J)$ and $\iota \in I$ are equivalent, we simply have

$$
\sum_{j \in J: j \geq n-k} \frac{\left(1-t_{0}\right)^{j+k-n}}{(j+k-n) !} P_{j}^{(\iota)}(1)=\frac{\left(1-t_{0}\right)^{k-\iota}}{(k-\iota) !} \mathbb{1}_{\{k \geq \iota\}}
$$

which immediately entails, by (4.73),

$$
Q_{i, t_{0}}^{(\iota)}\left(1-t_{0}\right)=0 \quad \text { for } \iota \in I
$$

Next, concerning $\tilde{P}_{j, t_{0}}^{(l)}$, we have

$$
\tilde{P}_{j, t_{0}}^{(()}(t)=P_{j}^{(\iota)}\left(t+t_{0}\right)-\sum_{i=0}^{n-1} P_{j}^{(i)}\left(t_{0}\right) Q_{i, t_{0}}^{(\iota)}(t)
$$


Choosing $t=0$, this gives, for $\iota \in\{0,1, \ldots, n-1\}$, since $Q_{i, t_{0}}^{(\iota)}(0)=\delta_{\iota, i}$,

$$
\tilde{P}_{j, t_{0}}^{(\iota)}(0)=P_{j}^{(\iota)}\left(t_{0}\right)-\sum_{i=0}^{n-1} P_{j}^{(i)}\left(t_{0}\right) Q_{i, t_{0}}^{(\iota)}(0)=0
$$

Choosing $t=1-t_{0}$, we have, for $\iota \in I$, since $P_{j}^{(\iota)}(1)=\delta_{\iota, n-j}$ and $Q_{i, t_{0}}^{(\iota)}\left(1-t_{0}\right)=0$,

$$
\widetilde{P}_{j, t_{0}}^{(\iota)}\left(1-t_{0}\right)=P_{j}^{(\iota)}(1)-\sum_{i=0}^{n-1} P_{j}^{(i)}\left(t_{0}\right) Q_{i, t_{0}}^{(\iota)}\left(1-t_{0}\right)=\delta_{\iota, n-j}
$$

The polynomials $\widetilde{P}_{j, t_{0}}$ (resp., $Q_{i, t_{0}}$ ) enjoy the same properties than the $P_{j}$ 's (resp., the $\widetilde{\psi}_{i}{ }^{\prime} \mathrm{s}$ ), regarding the successive derivatives, they can be deduced from these latter by a rescaling according as

$$
\widetilde{P}_{j, t_{0}}(t)=\left(1-t_{0}\right)^{n-j} P_{j}\left(\frac{t}{1-t_{0}}\right) \quad\left(\text { resp. } Q_{i, t_{0}}=\left(1-t_{0}\right)^{i} \widetilde{\psi}_{i}\left(\frac{t}{1-t_{0}}\right)\right)
$$

It is then easy to extract the identity in distribution below, by using the property of Gaussian conditioning:

$$
\left(\tilde{X}_{n}(t)-\sum_{j \in J} \tilde{P}_{j, t_{0}}(t) \tilde{X}_{j}\left(1-t_{0}\right)\right)_{t \in\left[0,1-t_{0}\right]} \stackrel{d}{=}\left(\tilde{X}_{n}(t) \mid \tilde{X}_{j}\left(1-t_{0}\right)=0, j \in J\right)_{t \in\left[0,1-t_{0}\right]} .
$$

Theorem 4.6 is established.

\section{Example: Bridges of Integrated Brownian Motion $(n=2)$}

Here, we have a look on the particular case where $n=2$ for which the corresponding process $\left(X_{n}(t)\right)_{t \in[0,1]}$ is nothing but integrated Brownian motion (the so-called Langevin process):

$$
X_{2}(t)=\int_{0}^{t} B(s) \mathrm{d} s
$$

The underlying Markov process is the so-called Kolmogorov diffusion $\left(X_{2}(t), X_{1}(t)\right)_{t \in[0,1]}$. All the associated conditioned processes that will be constructed are related to the equation $v^{(4)}(t)=u(t)$ with boundary value conditions at time $0: v(0)=v^{\prime}(0)=0$. There are four such processes:

(i) $\left(X_{2}(t)\right)_{t \in[0,1]}$ (integrated Brownian motion);

(ii) $\left(X_{2}(t) \mid X_{1}(1)=0\right)_{t \in[0,1]}$ (integrated Brownian bridge); 
(iii) $\left(X_{2}(t) \mid X_{2}(1)=0\right)_{t \in[0,1]}$ (bridge of integrated Brownian motion);

(iv) $\left(X_{2}(t) \mid X_{1}(1)=X_{2}(1)=0\right)_{t \in[0,1]}$ (another bridge of integrated Brownian motion).

On the other hand, when adding two boundary value conditions at time 1 to the foregoing equation, we find six boundary value problems: $v(1)=v^{\prime}(1)=0, v(1)=v^{\prime \prime}(1)=0, v(1)=$ $v^{\prime \prime \prime}(1)=0, v^{\prime}(1)=v^{\prime \prime}(1)=0, v^{\prime}(1)=v^{\prime \prime \prime}(1)=0, v^{\prime \prime}(1)=v^{\prime \prime \prime}(1)=0$. Actually, only four of them can be related to some Gaussian processes-the previously listed processes-in the sense of our work whereas two others cannot be.

For each process, we provide the covariance function, the representation by means of integrated Brownian motion subject to a random polynomial drift, the related boundary value conditions at 1 , and the decomposition related to the prediction problem. Since the computations are straightforward, we will omit them and we only report here the results.

For an account on integrated Brownian motion in relation with the present work, we refer the reader to, for example, [16] and references therein.

\subsection{Integrated Brownian Motion}

The process corresponding to the set $I=\emptyset$ is nothing but integrated Brownian motion:

$$
\left(X_{2}(t)\right)_{t \in[0,1]}=\left(\int_{0}^{t} B(s) \mathrm{d} s\right)_{t \in[0,1]}
$$

The covariance function is explicitly given by

$$
c(s, t)=\frac{1}{6}[s \wedge t]^{2}[3(s \vee t)-s \wedge t] .
$$

This process is related to the boundary value conditions at $1(J=\{2,3\}): v^{\prime \prime}(1)=v^{\prime \prime \prime}(1)=0$. The prediction property can be stated as follows:

$$
\left(X_{2}\left(t+t_{0}\right)\right)_{t \in\left[0,1-t_{0}\right]}=\left(\tilde{X}_{2}(t)+X_{2}\left(t_{0}\right)+t X_{1}\left(t_{0}\right)\right)_{t \in\left[0,1-t_{0}\right]} .
$$

\subsection{Integrated Brownian Bridge}

The process corresponding to the set $I=\{1\}$ is integrated Brownian bridge:

$$
(Y(t))_{t \in[0,1]}=\left(\int_{0}^{t} B(s) \mathrm{d} s \mid B(1)=0\right)_{t \in[0,1]}=\left(\int_{0}^{t} \beta(s) \mathrm{d} s\right)_{t \in[0,1]}=\left(X_{2}(t) \mid X_{1}(1)=0\right)_{t \in[0,1]} .
$$

This process can be represented as

$$
(Y(t))_{t \in[0,1]}=\left(X_{2}(t)-\frac{1}{2} t^{2} X_{1}(1)\right)_{t \in[0,1]}
$$


The covariance function is explicitly given by

$$
c(s, t)=\frac{1}{6}[s \wedge t]^{2}[3(s \vee t)-s \wedge t]-\frac{1}{4} s^{2} t^{2}
$$

The process $(Y(t))_{t \in[0,1]}$ is related to the boundary value conditions at $1(J=\{1,3\}): v^{\prime}(1)=$ $v^{\prime \prime \prime}(1)=0$. The prediction property says that

$$
\left(Y\left(t+t_{0}\right)\right)_{t \in\left[0,1-t_{0}\right]}=\left(\tilde{Y}_{t_{0}}(t)+Y\left(t_{0}\right)+\left(t-\frac{t^{2}}{2\left(1-t_{0}\right)}\right) Y^{\prime}\left(t_{0}\right)\right)_{t \in\left[0,1-t_{0}\right]} .
$$

\subsection{Bridge of Integrated Brownian Motion}

The process corresponding to the set $I=\{2\}$ is the bridge of integrated Brownian motion:

$$
(Y(t))_{t \in[0,1]}=\left(\int_{0}^{t} B(s) \mathrm{d} s \mid \int_{0}^{1} B(s) \mathrm{d} s=0\right)_{t \in[0,1]}=\left(X_{2}(t) \mid X_{2}(1)=0\right)_{t \in[0,1]}
$$

The bridge is understood as the process is pinned at its extremities: $Y(0)=Y(1)=0$. This process can be represented as

$$
(Y(t))_{t \in[0,1]}=\left(X_{2}(t)-\frac{1}{2} t^{2}(3-t) X_{2}(1)\right)_{t \in[0,1]}
$$

The covariance function is explicitly given by

$$
c(s, t)=\frac{1}{6}[s \wedge t]^{2}[3(s \vee t)-s \wedge t]-\frac{1}{12} s^{2} t^{2}(3-s)(3-t) .
$$

The process $(Y(t))_{t \in[0,1]}$ is related to the boundary value conditions at $1(J=\{0,2\}): v(1)=$ $v^{\prime \prime}(1)=0$. The prediction property says that

$$
\begin{aligned}
& \left(Y\left(t+t_{0}\right)\right)_{t \in\left[0,1-t_{0}\right]} \\
& \quad=\left(\tilde{Y}_{t_{0}}(t)+\frac{t^{3}-3\left(1-t_{0}\right) t^{2}+2\left(1-t_{0}\right)^{3}}{2\left(1-t_{0}\right)^{3}} Y\left(t_{0}\right)+\frac{t^{3}-3\left(1-t_{0}\right) t^{2}+2\left(1-t_{0}\right)^{2} t}{2\left(1-t_{0}\right)^{2}} Y^{\prime}\left(t_{0}\right)\right)_{t \in\left[0,1-t_{0}\right]} .
\end{aligned}
$$




\subsection{Other Bridge of Integrated Brownian Motion}

The process corresponding to the set $I=\{1,2\}$ is another bridge of integrated Brownian motion (actually of the two-dimensional Kolmogorov diffusion):

$$
(Y(t))_{t \in[0,1]}=\left(\int_{0}^{t} B(s) \mathrm{d} s \mid \int_{0}^{1} B(s) \mathrm{d} s=B(1)=0\right)_{t \in[0,1]}=\left(X_{2}(t) \mid X_{2}(1)=X_{1}(1)=0\right)_{t \in[0,1]} .
$$

The bridge here is understood as the process is pinned at its extremities together with its derivatives: $Y(0)=Y^{\prime}(0)=Y(1)=Y^{\prime}(1)=0$. This process can be represented as

$$
(Y(t))_{t \in[0,1]}=\left(X_{2}(t)-t^{2}(t-1) X_{1}(1)-t^{2}(3-2 t) X_{2}(1)\right)_{t \in[0,1]}
$$

The covariance function is explicitly given by

$$
c(s, t)=\frac{1}{6}[s \wedge t]^{2}[1-s \vee t]^{2}[3(s \vee t)-s \wedge t-2 s t] .
$$

The process $(Y(t))_{t \in[0,1]}$ is related to the boundary value conditions at $1(J=\{0,1\}): v(1)=$ $v^{\prime}(1)=0$. The prediction property says that

$$
\left(Y\left(t+t_{0}\right)\right)_{t \in\left[0,1-t_{0}\right]}=\left(\tilde{Y}_{t_{0}}(t)+\frac{t^{2}\left(t+t_{0}-1\right)}{\left(1-t_{0}\right)^{3}} Y\left(t_{0}\right)+\frac{t^{2}\left(3-3 t_{0}-2 t\right)}{\left(1-t_{0}\right)^{2}} Y^{\prime}\left(t_{0}\right)\right)_{t \in\left[0,1-t_{0}\right]} .
$$

\subsection{Two Counterexamples}

(i) The solution of the problem associated with the boundary value conditions $v(1)=v^{\prime \prime \prime}(1)=$ 0 (which corresponds to the set $I_{1}=\{0,3\}$ ) is given by

$$
v(t)=\int_{0}^{1} G_{1}(s, t) u(s) \mathrm{d} s, \quad t \in[0,1]
$$

where

$$
G_{1}(s, t)=\frac{1}{6}[s \wedge t]^{2}[3(s \vee t)-s \wedge t]+\frac{1}{6} s^{2} t^{2}(s-3)
$$

(ii) The solution of the problem associated with the boundary value conditions $v^{\prime}(1)=v^{\prime \prime}(1)=$ 0 (which corresponds to the set $I_{2}=\{1,2\}$ ) is given by

$$
v(t)=\int_{0}^{1} G_{2}(s, t) u(s) \mathrm{d} s, \quad t \in[0,1]
$$


where

$$
G_{2}(s, t)=\frac{1}{6}[s \wedge t]^{2}[3(s \vee t)-s \wedge t]+\frac{1}{6} s^{2} t^{2}(t-3)
$$

We can observe the relationships $G_{1}(s, t)=G_{2}(t, s)$ and $I_{2}=\{0,1,2,3\} \backslash\left(3-I_{1}\right)$. The Green functions $G_{1}$ and $G_{2}$ are not symmetric, so they cannot be viewed as the covariance functions of any Gaussian process. In the next section, we give an explanation of these observations.

\section{General Boundary Value Conditions}

In this last part, we address the problem of relating the general boundary value problem

$$
\left\{\begin{array}{l}
v^{(2 n)}=(-1)^{n} u \quad \text { on }[0,1], \\
v(0)=v^{\prime}(0)=\cdots=v^{(n-1)}(0)=0, \\
v^{\left(i_{1}\right)}(1)=v^{\left(i_{2}\right)}(1)=\cdots=v^{\left(i_{n}\right)}(1)=0,
\end{array}\right.
$$

for any indices $i_{1}, i_{2}, \ldots, i_{n}$ such that $0 \leq i_{1}<i_{2}<\cdots<i_{n} \leq 2 n-1$, to some possible Gaussian process. Set $I=\left\{i_{1}, i_{2}, \ldots, i_{n}\right\}$. We have noticed in Theorem 4.4 and Remark 4.5 that, when $I$ satisfies the relationship $2 n-1-I=\{0,1, \ldots, 2 n-1\} \backslash I$, the system (6.1) admits a unique solution. We proved this fact by computing an energy integral. Actually, this fact holds for any set of indices I; see Lemma A.1.

Our aim is to characterize the set of indices $I$ for which the Green function of (6.1) can be viewed as the covariance function of a Gaussian process. A necessary condition for a function of two variables to be the covariance function of a Gaussian process is that it must be symmetric. So, we characterize the set of indices $I$ for which the Green function of (6.1) is symmetric and we will see that in this case this function is a covariance function. For this, we make use of general results concerning the relationships between boundary value problems and symmetric linear operators in Hilbert spaces. Since our results below are direct applications of Theorem 2 in Section 18.2 of [17, Chapter V], which describes the general form of boundary conditions corresponding to the self-adjoint extensions of standard symmetric operator, we only state them without any proof. We refer the reader to a draft [18] for more details and self-contained proofs.

\subsection{Representation of the Solution}

We first write out a representation for the Green function of (6.1).

Theorem 6.1. The boundary value problem (6.1) has a unique solution. The corresponding Green function admits the following representation, for $s, t \in[0,1]$ :

$$
G_{I}(s, t)=(-1)^{n} \mathbb{1}_{\{s \leq t\}} \frac{(t-s)^{2 n-1}}{(2 n-1) !}-(-1)^{n} \sum_{\iota \in I} \frac{(1-s)^{2 n-1-\iota}}{(2 n-1-\imath) !} R_{I, t}(t),
$$


Table 2

\begin{tabular}{lcccccccc}
\hline Conditioning set $J$ & $\emptyset$ & $\{1\}$ & $\{2\}$ & $\{3\}$ & $\{1,2\}$ & $\{1,3\}$ & $\{2,3\}$ & $\{1,2,3\}$ \\
\hline Differentiating set $I$ & $\{3,4,5\}$ & $\{2,4,5\}$ & $\{1,3,5\}$ & $\{0,3,4\}$ & $\{1,2,5\}$ & $\{0,2,4\}$ & $\{0,1,3\}$ & $\{0,1,2\}$ \\
\hline
\end{tabular}

Table 3

\begin{tabular}{lllllll}
\hline Differentiating set $I_{1}$ & $\{0,1,4\}$ & $\{0,1,5\}$ & $\{0,2,5\}$ & $\{0,3,5\}$ & $\{0,4,5\}$ & $\{1,4,5\}$ \\
\hline Differentiating set $I_{2}$ & $\{0,2,3\}$ & $\{1,2,3\}$ & $\{1,2,4\}$ & $\{1,3,4\}$ & $\{2,3,4\}$ & $\{2,3,5\}$ \\
\hline
\end{tabular}

where the $R_{I, l}, \iota \in I$, are Hermite interpolation polynomials satisfying

$$
\left\{\begin{array}{l}
R_{I, l}^{(2 n)}=0, \\
R_{I, l}^{(i)}(0)=0 \quad \text { for } i \in\{0,1, \ldots, n-1\} \\
R_{I, l}^{(i)}(1)=\delta_{\iota, i} \quad \text { for } i \in I .
\end{array}\right.
$$

Remark 6.2. Conditions (6.3) completely characterize the polynomials $R_{I, \iota} \iota \in I$ (see Lemma A.1 in the appendix).

A necessary condition for $G_{I}$ to be the covariance function of a Gaussian process is that it must be symmetric: $G_{I}(s, t)=G_{I}(t, s)$ for any $s, t \in[0,1]$. The theorem below asserts that if the set of indices $I$ is not of the form displayed in the preamble of Section 4 , that is, $I \neq\{0,1, \ldots, 2 n-1\} \backslash(2 n-1-I)$, the Green function $G_{I}$ is not symmetric and consequently this function cannot be viewed as a covariance function, that is, we cannot relate the boundary value problem (6.1) to any Gaussian process.

Theorem 6.3. The Green function $G_{I}$ is symmetric (and it corresponds to a covariance function) if and only if the set of indices $I$ satisfies $2 n-1-I=\{0,1, \ldots, 2 n-1\} \backslash I$.

\subsection{Example: Bridges of Twice Integrated Brownian Motion $(n=3)$}

Here, we have a look on the particular case where $n=3$ for which the corresponding process $\left(X_{n}(t)\right)_{t \in[0,1]}$ is the twice integrated Brownian motion:

$$
X_{3}(t)=\int_{0}^{t}(t-s) B(s) \mathrm{d} s=\int_{0}^{t}\left(\int_{0}^{s_{2}} B\left(s_{1}\right) \mathrm{d} s_{1}\right) \mathrm{d} s_{2}
$$

All the associated conditioned processes that can be constructed are related to the equation $v^{(6)}(t)=-u(t)$ with boundary value conditions at time $0: v(0)=v^{\prime}(0)=v^{\prime \prime}(0)=0$. There are $2^{3}=8$ such processes. Since the computations are tedious and the explicit results are cumbersome, we only report the correspondence between bridges and boundary value conditions at time 1 through the sets of indices $I$ and $J$. These are written in Table 2.

The Green functions related to the other sets cannot be related to some Gaussian processes. The sets are written in Table 3 with the correspondence $I_{2}=\{0,1,2,3,4,5\} \backslash\left(5-I_{1}\right)$. 


\subsection{Example: Bridges of Thrice Integrated Brownian Motion $(n=4)$}

For $n=4$, only the $2^{4}=16$ following differentiating sets can be related to bridges:

$\{0,1,2,3\},\{0,1,2,4\},\{0,1,3,5\},\{0,1,4,5\},\{0,2,3,6\},\{0,2,4,6\},\{0,3,5,6\},\{0,4,5,6\}$,

$\{1,2,3,7\},\{1,2,4,7\},\{1,3,5,7\},\{1,4,5,7\},\{2,3,6,7\},\{2,4,6,7\},\{3,5,6,7\},\{4,5,6,7\}$.

\section{Appendix}

\section{Hermite Interpolation Polynomials}

Lemma A.1. Let $a_{i}, i \in\{0,1, \ldots, n-1\}$, and $b_{i}, i \in I$, be real numbers. There exists a unique polynomial P such that

$$
\left\{\begin{array}{l}
P^{(2 n)}=0, \\
P^{(i)}(0)=a_{i} \quad \text { for } i \in\{0,1, \ldots, n-1\} \\
P^{(i)}(1)=b_{i} \quad \text { for } i \in I .
\end{array}\right.
$$

Remark A.2. Conditions (A.1) characterize the Hermite interpolation polynomial at points 0 and 1 with given values of the successive derivatives at 0 up to order $n-1$ and given values of the derivatives at 1 with selected orders in $I$. When $I \neq\{0,1, \ldots, n-1\}$, these polynomials differ from the usual Hermite interpolation polynomials which involve the successive derivatives at certain points progressively from zero order up to certain orders.

Proof. We look for polynomials $P$ in the form $P(t)=\sum_{j=0}^{2 n-1} c_{j} t^{j} / j !$. We have

$$
P^{(i)}(t)=\sum_{j=0}^{2 n-1} c_{j} \frac{t^{j-i}}{(j-i) !}
$$

We will adopt the convention $1 /[(j-i) !]=0$ for $i>j$. Conditions (A.1) yield the linear system (with the convention that $i$ and $j$ denote, respectively the raw and column indices)

$$
\left\{\begin{array}{l}
c_{i}=a_{i} \quad \text { if } i \in\{0,1, \ldots, n-1\}, \\
\sum_{j=0}^{2 n-1} \frac{c_{j}}{(j-i) !}=b_{i} \quad \text { if } i \in I .
\end{array}\right.
$$


The $(2 n) \times(2 n)$ matrix of this system writes

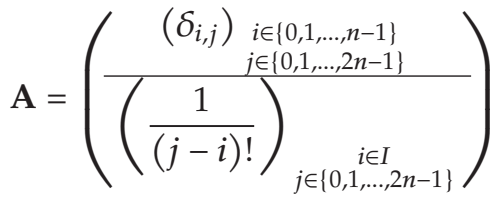

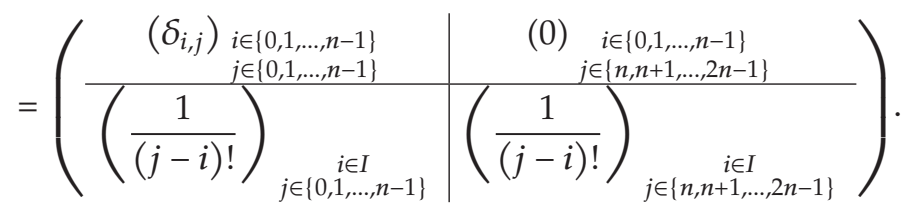

Proving the statement of Lemma A.1 is equivalent to proving that the matrix $\mathbf{A}$ is regular. In view of the form of $\mathbf{A}$ as a bloc-matrix, we see, since the north-west bloc is the unit matrix and the north-east bloc is the null matrix, that this is equivalent to proving that the south-east bloc of $\mathbf{A}$ is regular. Let us call this latter $\mathbf{A}_{0}$ and label its columns $\mathbf{C}_{j}^{(0)}, j \in\{0,1, \ldots, n-1\}$ :

$$
\mathbf{A}_{0}=\left(\frac{1}{(n+j-i) !}\right) \underset{\substack{i \in I \\
j \in\{0,1, \ldots, n-1\}}}{ }=\left(\begin{array}{llll}
\mathbf{C}_{0}^{(0)} & \mathbf{C}_{1}^{(0)} & \cdots & \mathbf{C}_{n-1}^{(0)}
\end{array}\right)
$$

For proving that $\mathbf{A}_{0}$ is regular, we factorize $\mathbf{A}_{0}$ into the product of two regular triangular matrices. The method consists in performing several transformations on the columns of $\mathbf{A}_{0}$ which do not affect its rank. We provide in this way an algorithm leading to an LUfactorization of $\mathbf{A}_{0}$, where $\mathbf{L}$ is a lower triangular matrix and $\mathbf{U}$ is an upper triangular matrix with no vanishing diagonal term.

We begin by performing the transformation

$$
\mathbf{C}_{j}^{(1)}= \begin{cases}\mathbf{C}_{j}^{(0)} & \text { if } j=0, \\ \mathbf{C}_{j}^{(0)}-\frac{\mathbf{C}_{j-1}^{(0)}}{n+j-i_{1}} & \text { if } j \in\{1,2, \ldots, n-1\} .\end{cases}
$$

The generic term of the column $\mathbf{C}_{j}^{(1)}$, for $j \in\{1,2, \ldots, n-1\}$, is

$$
\frac{1}{(n+j-i) !}-\frac{1}{n+j-i_{1}} \frac{1}{(n+j-i-1) !}=\frac{i-i_{1}}{n+j-i_{1}} \frac{1}{(n+j-i) !}
$$

This transformation supplies a matrix $\mathbf{A}_{1}$ with columns $\mathbf{C}_{j}^{(1)}, j \in\{0,1, \ldots, n-1\}$, which writes

$$
\mathbf{A}_{1}=\left(\begin{array}{llll}
\mathbf{C}_{0}^{(1)} & \mathbf{C}_{1}^{(1)} & \ldots & \mathbf{C}_{n-1}^{(1)}
\end{array}\right)=\left(\left(\frac{1}{(n-i) !}\right)_{i \in I} \mid\left(\frac{i-i_{1}}{n+j-i_{1}} \frac{1}{(n+j-i) !}\right)_{\substack{j \in\{1,2, \ldots, n-1\} \\
i \in I}}\right)
$$


We have written

$$
\mathbf{A}_{1}=\mathbf{A}_{0} \mathbf{U}_{1}
$$

where $\mathbf{U}_{1}$ is the triangular matrix with a diagonal made of 1 below:

$$
\mathbf{U}_{1}=\left(\delta_{i, j}-\frac{\delta_{i, j-1} \mathbb{1}_{\{j \geq 1\}}}{n+j-i_{1}}\right)_{0 \leq i, j \leq n-1}
$$

We now perform the transformation

$$
\mathbf{C}_{j}^{(2)}= \begin{cases}\mathbf{C}_{j}^{(1)} & \text { if } j \in\{0,1\}, \\ \mathbf{C}_{j}^{(1)}-\frac{n+j-i_{1}-1}{n+j-i_{1}} \frac{\mathbf{C}_{j-1}^{(1)}}{n+j-i_{2}} & \text { if } j \in\{2,3, \ldots, n-1\} .\end{cases}
$$

The generic term of the column $\mathbf{C}_{j}^{(2)}$, for $j \in\{2,3, \ldots, n-1\}$, is

$$
\begin{gathered}
\frac{i-i_{1}}{n+j-i_{1}} \frac{1}{(n+j-i) !}-\frac{i-i_{1}}{\left(n+j-i_{1}\right)\left(n+j-i_{2}\right)} \frac{1}{(n+j-i-1) !} \\
=\frac{\left(i-i_{1}\right)\left(i-i_{2}\right)}{\left(n+j-i_{1}\right)\left(n+j-i_{2}\right)} \frac{1}{(n+j-i) !} .
\end{gathered}
$$

This transformation supplies a matrix $\mathbf{A}_{2}$ with columns $\mathbf{C}_{j}^{(2)}, j \in\{0,1, \ldots, n-1\}$, which writes

$$
\begin{aligned}
& \mathbf{A}_{2}=\left(\begin{array}{llll}
\mathbf{C}_{0}^{(2)} & \mathbf{C}_{1}^{(2)} & \cdots & \mathbf{C}_{n-1}^{(2)}
\end{array}\right) \\
& =\left(\left(\frac{1}{(n-i) !}\right)_{i \in I} \mid\left(\frac{i-i_{1}}{n+1-i_{1}} \frac{1}{(n+1-i) !}\right)_{i \in I}\right. \\
& \left.\mid\left(\frac{\left(i-i_{1}\right)\left(i-i_{2}\right)}{\left(n+j-i_{1}\right)\left(n+j-i_{2}\right)} \frac{1}{(n+j-i) !}\right)_{\substack{i \in I \\
j \in\{2,3, \ldots, n-1\}}}\right) .
\end{aligned}
$$

We have written

$$
\mathbf{A}_{2}=\mathbf{A}_{1} \mathbf{U}_{2}=\mathbf{A}_{0} \mathbf{U}_{1} \mathbf{U}_{2}
$$

where $\mathbf{U}_{2}$ is the triangular matrix with a diagonal made of 1 :

$$
\mathbf{U}_{2}=\left(\delta_{i, j}-\frac{n+j-i_{1}-1}{\left(n+j-i_{1}\right)\left(n+j-i_{2}\right)} \delta_{i, j-1} \mathbb{1}_{\{j \geq 2\}}\right)_{0 \leq i, j \leq n-1} .
$$


In a recursive manner, we easily see that we can construct a sequence of matrices $\mathbf{A}_{k}, \mathbf{U}_{k}$, $k \in\{1,2, \ldots, n-1\}$, such that $\mathbf{A}_{k}=\mathbf{A}_{k-1} \mathbf{U}_{k}$, where $\mathbf{U}_{k}$ is the triangular matrix with a diagonal made of 1 :

$$
\begin{aligned}
& \mathbf{U}_{k}=\left(\delta_{i, j}-\frac{\left(n+j-i_{1}-1\right) \cdots\left(n+j-i_{k-1}-1\right)}{\left(n+j-i_{1}\right) \cdots\left(n+j-i_{k}\right)} \delta_{i, j-1} \mathbb{1}_{\{j \geq k\}}\right)_{0 \leq i, j \leq n-1}, \\
& \mathbf{A}_{k}=\left(\left(\frac{1}{(n-i) !}\right)_{i \in I}\left|\left(\frac{i-i_{1}}{n+1-i_{1}} \frac{1}{(n+1-i) !}\right)_{i \in I}\right| \ldots\right. \\
& \mid\left(\frac{\left(i-i_{1}\right) \cdots\left(i-i_{k-1}\right)}{\left(n+k-1-i_{1}\right) \cdots\left(n+k-1-i_{k-1}\right)} \frac{1}{(n+k-1-i) !}\right)_{i \in I} \\
& \left.\mid\left(\frac{\left(i-i_{1}\right) \cdots\left(i-i_{k}\right)}{\left(n+j-i_{1}\right) \cdots\left(n+j-i_{k}\right)} \frac{1}{(n+j-i) !}\right) \underset{j \in\{k, \ldots, n-1\}}{i \in I}\right) \text {. }
\end{aligned}
$$

We finally obtain, since all the $\mathbf{U}_{k} k \in\{1,2, \ldots, n-1\}$, are regular, that

$$
\mathbf{A}_{0}=\mathbf{A}_{n-1} \mathbf{U}_{n-1}^{-1} \cdots \mathbf{U}_{1}^{-1}=\mathbf{L} \mathbf{U}
$$

with $\mathbf{U}=\mathbf{U}_{n-1}^{-1} \cdots \mathbf{U}_{1}^{-1}$ and

$$
\begin{aligned}
\mathbf{L}= & \mathbf{A}_{n-1} \\
= & \left(\left(\frac{1}{(n-i) !}\right)_{i \in I}\left|\left(\frac{i-i_{1}}{n+1-i_{1}} \frac{1}{(n+1-i) !}\right)_{i \in I}\right| \ldots\right. \\
& \left.\mid\left(\frac{\left(i-i_{1}\right) \cdots\left(i-i_{n-1}\right)}{\left(2 n-1-i_{1}\right) \cdots\left(2 n-1-i_{n-1}\right)} \frac{1}{(2 n-1-i) !}\right)_{i \in I}\right) .
\end{aligned}
$$

It is clear that the matrices $\mathbf{U}$ and $\mathbf{L}$ are triangular and regular, and then $\mathbf{A}_{0}$ (and $\mathbf{A}$ ) is also regular. Moreover, the inverse of $\mathbf{A}_{0}$ can be computed as

$$
\mathbf{A}_{0}^{-1}=\mathbf{U}^{-1} \mathbf{L}^{-1}=\mathbf{U}_{1} \cdots \mathbf{U}_{n-1} \mathbf{L}^{-1} .
$$

The proof of Lemma A.1 is finished.

\section{References}

[1] A. Lachal, "Study of some new integrated statistics: computation of Bahadur efficiency, relation with non-standard boundary value problems," Mathematical Methods of Statistics, vol. 10, no. 1, pp. 73-104, 2001.

[2] A. Lachal, "Bridges of certain Wiener integrals. Prediction properties, relation with polynomial interpolation and differential equations. Application to goodness-of-fit testing, " in Bolyai Society Mathematical Studies X. Limit Theorems, Balatonlelle (Hungary), 1999, pp. 1-51, Budapest, Hungary, 2002.

[3] N. Henze and Y. Y. Nikitin, "A new approach to goodness-of-fit testing based on the integrated empirical process," Journal of Nonparametric Statistics, vol. 12, no. 3, pp. 391-416, 2000. 
[4] N. Henze and Ya. Yu. Nikitin, "Watson-type goodness-of-fit tests based on the integrated empirical process," Mathematical Methods of Statistics, vol. 11, no. 2, pp. 183-202, 2002.

[5] C. L. Dolph and M. A. Woodbury, "On the relation between Green's functions and covariances of certain stochastic processes and its application to unbiased linear prediction," Transactions of the American Mathematical Society, vol. 72, pp. 519-550, 1952.

[6] L. Carraro, "Étude de la covariance de quelques processus Gaussiens en liaison avec la propriété de Markov," Stochastic Processes and Their Applications, vol. 35, no. 2, pp. 251-265, 1990.

[7] X. Chen and W. V. Li, "Quadratic functionals and small ball probabilities for the m-fold integrated brownian motion," Annals of Probability, vol. 31, no. 2, pp. 1052-1077, 2003.

[8] A. Lachal, "Regular points for the successive primitives of Brownian motion," Journal of Mathematics of Kyoto University, vol. 37, no. 1, pp. 99-119, 1997.

[9] A. Lachal, "Local asymptotic classes for the successive primitives of Brownian motion," Annals of Probability, vol. 25, no. 4, pp. 1712-1734, 1997.

[10] Z. Lin, "Path properties of the primitives of a Brownian motion," Journal of the Australian Mathematical Society, vol. 70, no. 1, pp. 119-133, 2001.

[11] A. I. Nazarov, "Exact $L_{2}$-small ball asymptotics of Gaussian processes and the spectrum of boundaryvalue problems," Journal of Theoretical Probability, vol. 22, no. 3, pp. 640-665, 2009.

[12] A. I. Nazarov and Y. Y. Nikitin, "Exact $L_{2}$-small ball behavior of integrated Gaussian processes and spectral asymptotics of boundary value problems," Probability Theory and Related Fields, vol. 129, no. 4, pp. 469-494, 2004.

[13] R. Zhang and Z. Lin, "A functional LIL for m-Fold integrated Brownian motion," Chinese Annals of Mathematics. Series B, vol. 27, no. 4, pp. 459-472, 2006.

[14] G. Wahba, "Improper priors, spline smoothing and the problem of guarding against model error in regression," Journal of the Royal Statistical Society. Series B, vol. 40, no. 3, pp. 364-372, 1978.

[15] G. Wahba, "Bayesian "confidence intervals" for the cross-validated smoothing spline," Journal of the Royal Statistical Society. Series B, vol. 45, no. 1, pp. 133-150, 1983.

[16] A. Lachal, "Sur la distribution de certaines fonctionnelles de l'intégrale du mouvement Brownien avec dérives parabolique et cubique," Communications on Pure and Applied Mathematics, vol. 49, no. 12, pp. 1299-1338, 1996.

[17] I. Naimark, Linear Differential Operators. Part II: Linear Differential Operators in Hilbert Spaces, Frederick Ungar Publishing, New York, NY, USA, 1968.

[18] A. Lachal, "A class of bridges of iterated integrals of Brownian motion related to various boundary value problems involving the one-dimensional polyharmonic operator," http://arxiv.org/abs/ 1108.2132. 


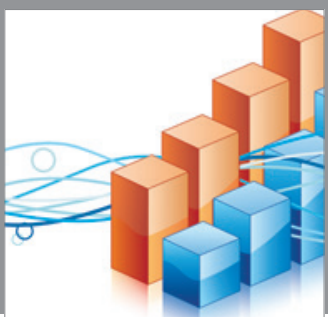

Advances in

Operations Research

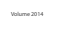

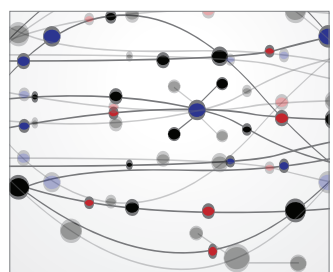

\section{The Scientific} World Journal
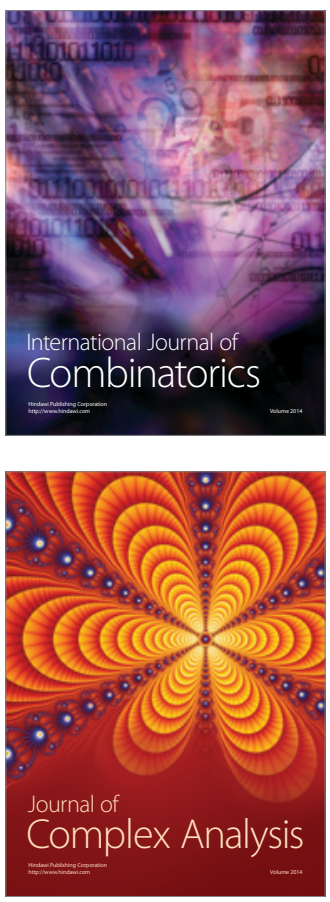

International Journal of

Mathematics and

Mathematical

Sciences
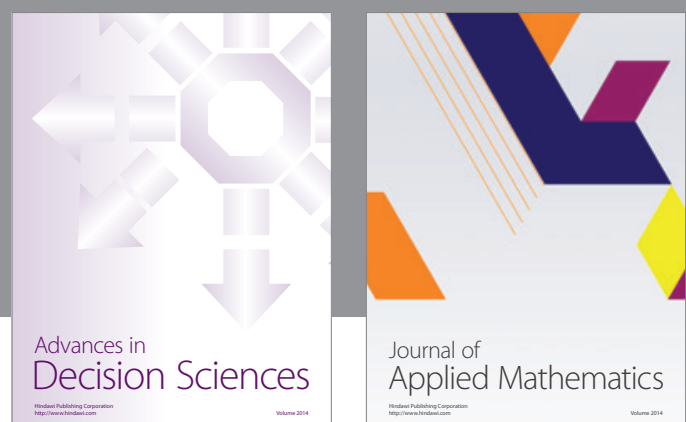

Journal of

Applied Mathematics
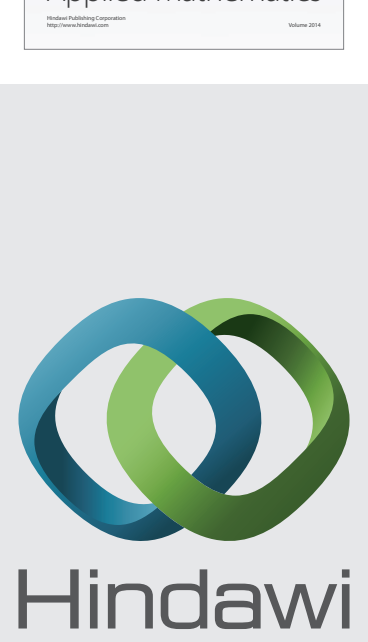

Submit your manuscripts at http://www.hindawi.com
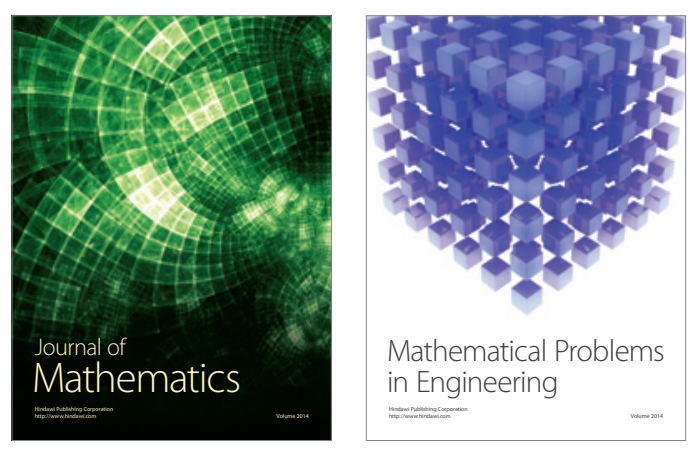

Mathematical Problems in Engineering
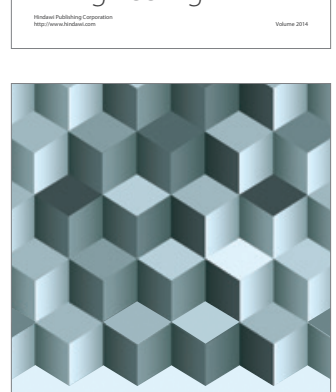

Journal of

Function Spaces
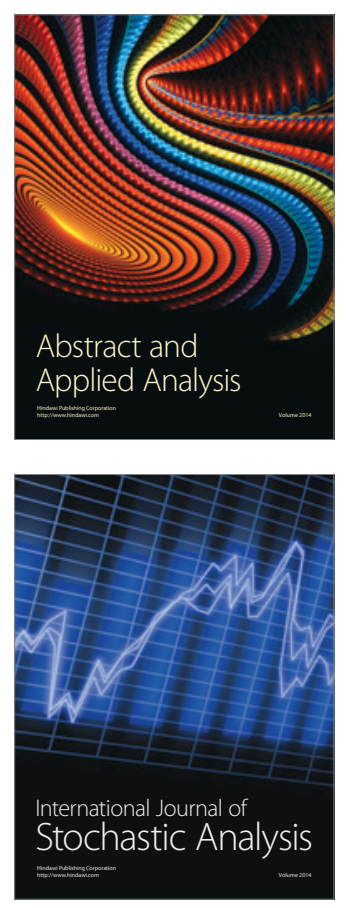

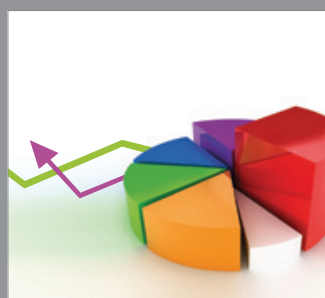

ournal of

Probability and Statistics

Promensencen
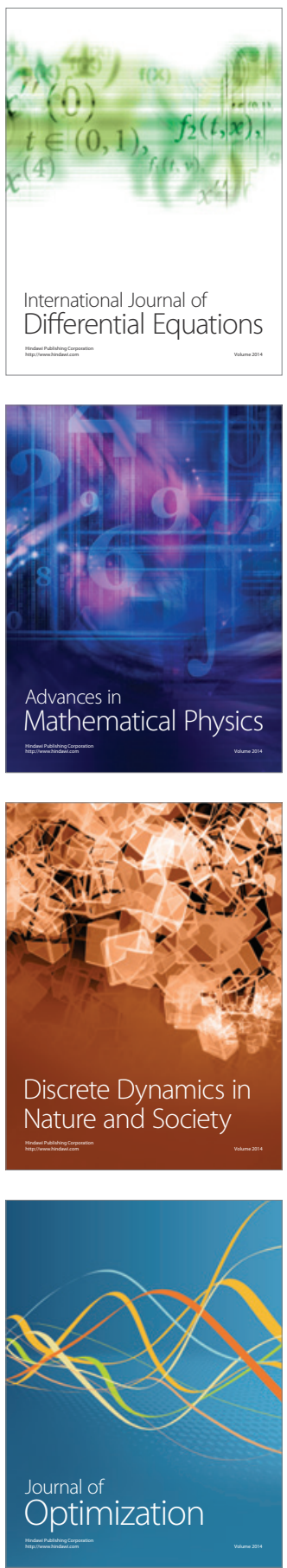